\title{
Changing look AGN Mrk 590: Broad line region and black hole mass from photometric reverberation mapping
}

\author{
Amit Kumar Mandal ${ }^{1,2 \star}$, Malte Schramm³, Suvendu Rakshit ${ }^{4}$, C. S. Stalin ${ }^{1}$, \\ Bovornpratch Vijarnwannaluk ${ }^{5}$, Wiphu Rujopakarn ${ }^{6,7}$, Saran Poshyachinda ${ }^{7}$, \\ Vladimir V. Kouprianov ${ }^{8,9}$, Joshua B. Haislip ${ }^{8}$, Daniel E. Reichart ${ }^{8}$, \\ Ram Sagar ${ }^{1}$ and Blesson Mathew ${ }^{2}$ \\ ${ }^{1}$ Indian Institute of Astrophysics, Block II, Koramangala, Bangalore, 560 034, India \\ ${ }^{2}$ Department of Physics, CHRIST (Deemed to be University), Hosur Road, Bangalore 560 029, India \\ ${ }^{3}$ Graduate school of Science and Engineering, Saitama Univ. 255 Shimo-Okubo, Sakura-ku, Saitama City, Saitama 338-8570, JAPAN \\ ${ }^{4}$ Aryabhatta Research Institute of Observational Sciences, Manora Peak, Nainital 263002, India \\ 5 Astronomical Institute, Tohoku University, 6-3 Aramaki, Aoba-ku Sendai, Miyagi 980-8578, Japan \\ 6 Department of Physics, Faculty of Science, Chulalongkorn University, 254 Phayathai Road, Pathumwan, Bangkok 10330, Thailand \\ 7 National Astronomical Research Institute of Thailand, 260 Moo 4, T. Donkaew, A. Maerim, Chiangmai, 50180 Thailand \\ ${ }^{8}$ Department of Physics and Astronomy, University of North Carolina at Chapel Hill, Campus Box 3255, Chapel Hill, NC 27599-3255 \\ 9 Central (Pulkovo) Observatory of the Russian Academy of Sciences 196140,65/1 Pulkovskoye Ave., Saint Petersburg, Russia
}

Accepted: 2021 October 5; Received: 2021 October 1; in original form: 2021 June 21

\begin{abstract}
We present the results of photometric reverberation mapping observations on the changing look active galactic nucleus Mrk 590 at $\mathrm{z}=0.026$. The observations were carried out from July to December, 2018 using broad band B, R and narrow band $\mathrm{H} \alpha$ and S II filters. B-band traces the continuum emission from the accretion disk, R-band encompasses both the continuum emission from the accretion disk and the redshifted $\mathrm{H} \alpha$ line from the broad line region (BLR), S II band contains the redshifted $\mathrm{H} \alpha$ emission and the $\mathrm{H} \alpha$ band traces the continuum emission underneath the S II band. All the light curves showed strong variation with a fractional root-mean-square variation of $0.132 \pm 0.001$ in $B$-band and $0.321 \pm 0.001$ in $\mathrm{H} \alpha$ line. From cross-correlation function analysis, we obtained a delayed response of $\mathrm{H} \alpha$ line emission to the optical B-band continuum emission of $21.44_{-2.11}^{+1.49}$ days in the rest-frame of the source, corresponding to a linear size of the BLR of $0.018 \mathrm{pc}$. This is consistent with previous estimates using $\mathrm{H} \beta$. By combining the BLR size with the $\mathrm{H} \alpha$ line full width at half maximum of $6478 \pm 240 \mathrm{~km} \mathrm{~s}^{-1}$ measured from a single-epoch spectrum obtained with the Subaru telescope, we derived a black hole mass of $1.96_{-0.21}^{+0.15} \times 10^{8} M_{\odot}$.
\end{abstract}

Key words: Galaxies, galaxies: active $<$ Galaxies, galaxies: individual: $\ldots<$ Galaxies, (galaxies:) quasars: emission lines < Galaxies, galaxies: Seyfert $<$ Galaxies

\section{INTRODUCTION}

Active Galactic Nuclei (AGN) are powered by accretion of matter onto the supermassive black hole $\left(\mathrm{SMBH} ; 10^{6}-10^{10}\right.$ $\left.\mathrm{M}_{\odot}\right)$ at the center of galaxies producing radiation covering the entire accessible electromagnetic spectrum. There is significant emission in the ultraviolet (UV)-optical blue continua from the accretion disk surrounding the SMBH in AGN (Lynden-Bell 1969; Salpeter 1964). The broad emis-

* E-mail: amitkumar@iiap.res.in sion lines are produced due to the thermal re-ionization of $\mathrm{UV} /$ optical continuum in the broad line region (BLR) that lies outside the accretion disk. According to the unified scheme of AGN, an obscuring torus produces the infrared (IR) thermal emission from the hot dust characterized by the sublimation temperature $\mathrm{T}_{\text {sub }}$ at which the dust particle in radiative equilibrium with the disk UV radiation, sublimates. For graphite grains the sublimation temperature is $\mathrm{T}_{\text {sub }} \sim 1700-2000 \mathrm{~K}$ (Minezaki et al. 2004; Suganuma et al. 2006; Kishimoto et al. 2007; Koshida et al. 2014). The central part of AGN is too compact to resolve using conven- 
tional imaging techniques. Though Gravity Collaboration et al. (2018) observed 3C 273 using GRAVITY and found a mean BLR size of $145 \pm 35$ days using the Pa $\alpha$ line through interferometric technique, it is limited to only very bright and nearby AGN.

An indirect method to probe the BLR region in AGN is the technique of reverberation mapping (RM; Blandford \& McKee 1982; Peterson 1993) that relies on the intrinsic flux variability properties of AGN. BLR RM is based on measuring the time delay between the UV/optical continuum from the accretion disk and the broad emission line produced in the BLR. As of today, BLR reverberation has provided the size of BLR in more than 100 AGN yielding a linear relation between the radius of the BLR $\left(\mathrm{R}_{\mathrm{BLR}}\right)$ and the continuum luminosity $\left(\mathrm{L}_{5100}\right)$ at $5100 \AA$ (Bentz \& Katz 2015; Grier et al. 2017; Du et al. 2014; Wang et al. 2014) in the luminosity range between $10^{42}-10^{46} \mathrm{erg} \mathrm{s}^{-1}$. The $R_{B L R}-L$ relation and a single-epoch spectrum allow us to estimate black hole masses for a large number of AGN, but it is not clear if the same relation holds over a wide range of luminosity mainly due to the lack of measurements both at the low luminosity end $\left(L<10^{42} \mathrm{erg} \mathrm{s}^{-1}\right)$ and the high luminosity end $\left(L>10^{46} \mathrm{erg} \mathrm{s}^{-1}\right)$.

Recently, number of AGN have been discovered, which undergo a transition from Type 1 characterized by prominent broad-emission lines, to Type 1.8 or 1.9 , with no or weak broad $\mathrm{H} \alpha$ and/or $\mathrm{H} \beta$, or vice versa (LaMassa 2015). These objects are known as "changing-look" AGN, among which Mrk 590 is a well known object. Its optical spectrum obtained by Peterson et al. (1998b) showed strong broad $\mathrm{H} \beta$ emission line but it disappeared between 2006 - 2013 (Denney et al. 2014) when the continuum luminosity decreased by a factor of 100 indicating that the black hole accretion rate has significantly decreased. The disappearance of broad emission lines in the UV/optical spectrum made Mrk 590 to appear as Seyfert 1.9-2, where the only broad emission line visible in the optical spectrum is a weak component of $\mathrm{H} \alpha$ (Denney et al. 2014). However, recently, Raimundo et al. (2019) noticed the reappearance of optical broad emission lines in Mrk 590 after $\sim 10$ yr of absence, while, the AGN optical continuum flux is still $\sim 10$ times lower than that observed during the most luminous state in the 1990s. During the period 1990 - 1996 when Mrk 590 was in a bright state, $\mathrm{H} \beta$ BLR RM monitoring was carried out by Peterson et al. (1998b, 2004) and Bentz et al. (2009, 2013). The BLR lag based on $\mathrm{H} \beta$ line ranges from 19.5 days to 30 days (Bentz et al. 2013; Zu et al. 2011).

Recently, dust reverberation mapping (DRM) study of Mrk 590 by Kokubo \& Minezaki (2020) suggests that the inner radius of the dust torus decreased after a drastic drop in the UV-optical luminosity. They found the innermost dust torus size of $\sim 32$ light-days during the faint state in 2003 -2007 , which is comparable to the $\mathrm{H} \beta$ BLR radius of $\sim 26$ light-days obtained by RM observations during the bright state in 1990 - 1996. We monitored Mrk 590 during the period July 2018 to December 2018 using broad $B, R$, narrow $\mathrm{H} \alpha$ and S II bands to estimate the size of the BLR based on the time delay between the $\mathrm{H} \alpha$ line and the continuum. Furthermore, to obtain spectral information and calculate black hole mass, we obtained a single-epoch spectrum using the Subaru telescope on October 27, 2018. Our black hole mass measurement using $\mathrm{H} \alpha$ is a new addition to the exist- ing information on Mrk 590. The outline of the paper is as follows. In section 2 we describe the observation and data reduction process. The time series analysis is presented in section 3 and the results are discussed in section 4 with a summary in section 5 .

\section{OBSERVATION AND DATA REDUCTION}

\subsection{Observations}

The photometric observations in the optical broad $B$ and $R$ filters as well as narrow $\mathrm{H} \alpha$ and S II filters were performed from July 2018 to December 2018 using the $60 \mathrm{~cm}$ robotic telescope PROMPT8 located at the Cerro Tololo Inter-American Observatory (CTIO) operated as part of Skynet (see Martin et al. 2019, for some introduction of the network). The observation period covers a total of 169 days with an attempted daily cadence. Our final data set consists of $115,90,86$ and 83 epochs in $B, R, \mathrm{H} \alpha$ and SII, respectively, achieving an effective cadence of $\sim 1.5$ days in the Bband and $\sim 2$ days in SII. Gaps in the light-curves are mainly due to weather loss or moon distance which prevented observations. The B-band was given the highest priority for the Skynet scheduler as it defines our baseline for the AGN continuum which requires the best possible cadence. The CCD has an average readout noise and gain of 10.21 electrons and 2.2 electrons/ADU, respectively, with a pixel scale of 0.662 arcsec/pixel covering a FoV of $23^{\prime} \times 23^{\prime}$. We used a 9-point dither pattern with a dither length of 50 arcsec to minimize the effect of bad pixel and cosmic rays. The typical total exposure times in $B, R, \mathrm{H} \alpha$ and S II bands are about 240s, 240 s, 900 s and 900 s, respectively. Since calibration images were not available for each night, the calibration closest in time was assigned to the target observation epoch.

\subsection{Optical spectrum}

We obtained a single-epoch spectrum with an exposure time of 3600s using the High Dispersion Spectrograph (HDS; Noguchi et al. 2002) at the f/12.71 optical Nasmyth focus of the $8.2 \mathrm{~m}$ Subaru telescope on October 27, 2018 under poor weather conditions. We used a long slit with 1 arcsec width and the data were sampled by two detectors (blue and red CCDs) with $4100 \times 2248$ pixels with a $2 \times 2$ binning resulting in a spectral resolution of $\mathrm{R}=18,000$ with a $\mathrm{S} / \mathrm{N}$ of 10 in the continuum. The data was reduced using IRAF ${ }^{1}$ (Image Reduction and Analysis Facility) tools and a custom built application written in Python 2.7 making use of the available command language scripts in IRAF for overscan and non-linearty correction obtained from the HDS web site ${ }^{2}$. The spectrum covers a wavelength range of $4354-7138 \AA$ and shown in Fig. 1, where the $B, R, \mathrm{H} \alpha$ and $\mathrm{S}$ II filter transmission curves are over-plotted.

To measure the emission line parameters, the Subaru spectrum was first corrected for Galactic extinction using the Schlegel et al. (1998) map and the Milky Way extinction

1 IRAF is operated by the Association of Universities for Research in Astronomy, Inc., under cooperative agreement with the National Science Foundation

2 https://www.subarutelescope.org/Observing/Instruments/HDS/index.html 


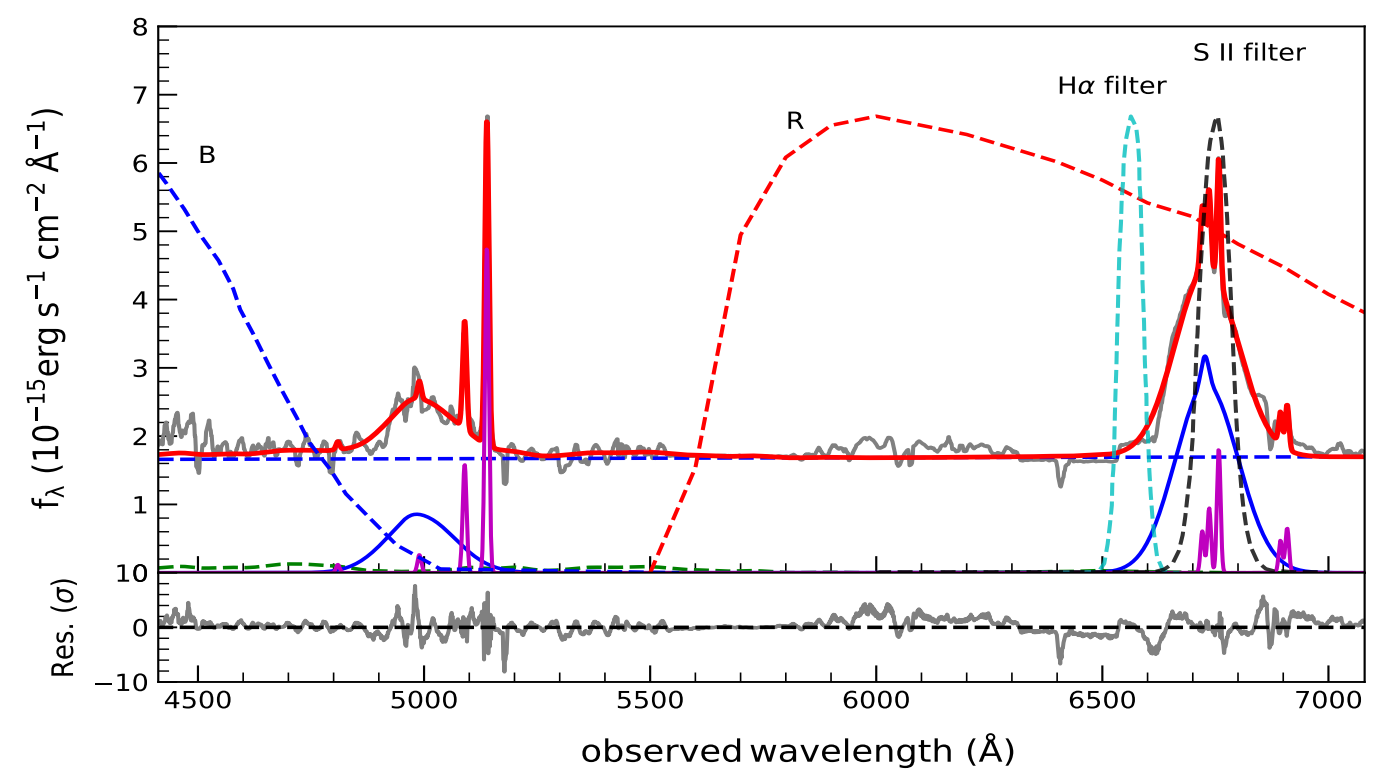

Figure 1. Subaru spectrum of Mrk 590. Top panel: The observed data (gray), best-fit model (red) and decomposed power-law continuum model (black dashed), broad line (blue), and narrow line (magenta) are shown. The broad $B, R$ and narrow H $\alpha$ and S II filters are over-plotted with limited x-axis for illustration. Bottom panel: Residual in the unit of flux uncertainty. The spectrum was smoothed by a 300 pixel box-car for visualization purpose.

Table 1. Spectral properties of Mrk 590. Columns are (1-2) FWHM and luminosity of H $\beta$ broad component, (3-4) FWHM and luminosity of [O III] $\lambda 5007,(5-6) \mathrm{FWHM}$ and luminosity of $\mathrm{H} \alpha$ broad component, (7) luminosity of $\mathrm{H} \alpha$ narrow component, and (8) luminosity at $5100 \AA$. The units of FWHM and luminosity are $\mathrm{km} \mathrm{s}^{-1}$ and $\mathrm{erg} \mathrm{s}^{-1}$, respectively. Line widths are not corrected for instrumental resolution.

\begin{tabular}{cccccccc}
\hline \hline $\begin{array}{c}\mathrm{FWHM}(\mathrm{H} \beta) \\
(1)\end{array}$ & $\log L(\mathrm{H} \beta)$ & $\mathrm{FWHM}([\mathrm{O} \mathrm{III}])$ & $\log L([\mathrm{O} \mathrm{III}])$ & $\mathrm{FWHM}(\mathrm{H} \alpha)$ & $\log L(\mathrm{H} \alpha)$ & $\log L\left(\mathrm{H} \alpha_{n}\right)$ & $\log \lambda L_{\lambda}(5100)$ \\
$(2)$ & $(3)$ & $(4)$ & $(5)$ & $(6)$ & $(7)$ & $(8)$ \\
\hline $10390 \pm 1718$ & $41.38 \pm 0.01$ & $592 \pm 7$ & $40.88 \pm 0.01$ & $6478 \pm 240$ & $41.86 \pm 0.01$ & $40.13 \pm 0.08$ & $43.12 \pm 0.01$ \\
\hline \hline
\end{tabular}

law of Fitzpatrick (1999) with $R_{V}=3.1$ and then moved to the rest-frame. A multi-component modeling was performed to estimate the emission line parameters. In the first step, we modeled the continuum, masking prominent emission lines, as a combination of a power-law $\left(f_{\lambda} \propto \lambda^{\alpha}\right)$ and an Fe II template from Boroson \& Green (1992) that accounts for the Fe II emission in AGN. The $\mathrm{H} \alpha$ and $\mathrm{H} \beta$ complexes of the best-fit continuum subtracted spectrum, was modeled using multiple Gaussians (e.g., Rakshit et al. 2020); upto three Gaussians for the broad lines and a single Gaussian for the narrow lines. The best-fit model is shown in Fig. 1 and the parameters are given in Table 1.

The broad B-band effectively traces the AGN continuum as the contribution of the higher order Balmer lines is negligible. The broad R-band contains the redshifted $\mathrm{H} \alpha$ line and the continuum flux from the accretion disk. The narrow S II band traces a large part of the redshifted $\mathrm{H} \alpha$ (see Fig. 1) line and thus the S II narrow band is mostly dominated by the response of the emission line clouds in the BLR. The AGN continuum underneath the emission line is traced by the narrow $\mathrm{H} \alpha$ band. Therefore, the pure emission line flux can be estimated by subtracting the narrow band $\mathrm{H} \alpha$ flux from the narrow band S II flux. Also, $\mathrm{H} \alpha$ emission line fluxes can be obtained from the R-band observations, however, continuum subtraction from observed R-band flux is a difficult task considering the larger accretion disk contribution compared to the narrow S II band.

\subsection{Data reduction}

We used IRAF to reduce all the broad $B$ and $R$, and narrow $\mathrm{H} \alpha$ and S II-band data. We followed the standard procedures for image reduction, such as bias and dark subtraction and flat-fielding.

The daofind task available in IRAF was used to detect the objects in the preprocessed images. Aperture photometry was performed using the phot task in IRAF. The final instrumental magnitudes were obtained using the method of curve of growth (COG; Mandal et al. 2021). We obtained the instrumental magnitudes in several concentric apertures centered on few comparison stars with good signal to noise ratio $(\mathrm{S} / \mathrm{N})$ and, present on the same $\mathrm{CCD}$ frame. The COG was produced by plotting the instrumental magnitudes obtained from different apertures against their corresponding aperture radii as shown in Fig. 2. The growth curve increases with aperture till the aperture is large enough to capture 


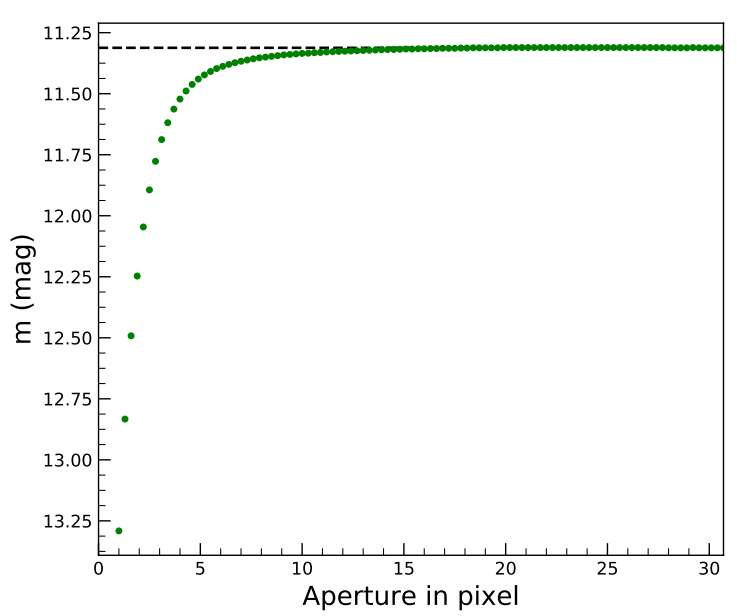

Figure 2. Growth curve for a comparison star in S II-band. The dashed black line is the best fit line that uses the points with aperture sizes between 5 and 8 times the FWHM.
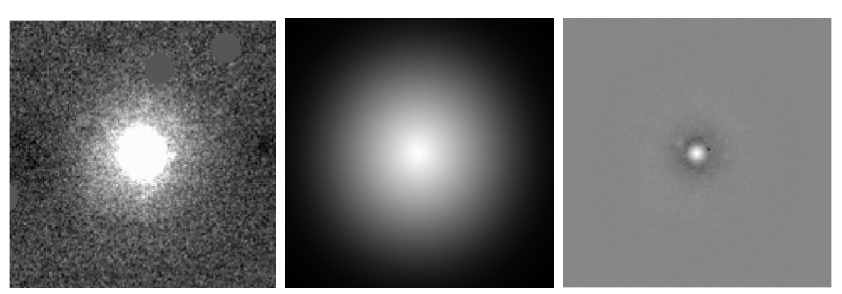

Figure 3. Observed S II-band image of Mrk 590 (left). Modeled galaxy image (middle) and residual image containing the AGN at the center (right) obtained from GALFIT. The modeled and residual images are displayed in logarithmic scale. The field of view of each image is $1.8^{\prime} \times 1.8^{\prime}$.

most of the flux from the source before gradually merging with the background. We fit a straight line based on the method of least squares to the portion of the growth curve with apertures between 5 and 8 times the FWHM of the comparison stars, where the COG merges with the background. The intercept of the fitted line (dashed black line in Fig. 2) was taken as the desired instrumental magnitude of the point source. This method was applied to each epoch to get the instrumental magnitudes of the comparison stars present in the CCD frames for all the bands.

\subsection{Correction for the host-galaxy contribution}

The host galaxy is prominently seen in the observed images of Mrk 590 in all the filters (see the left panel of Fig. 3 for S II filter). To remove the host-galaxy contribution from the total flux, we used the two dimensional image-decomposition program GALFIT developed by Peng et al. (2002, 2010). We combined several frames of different epochs with good seeing condition covering the whole span of our monitoring period to obtain a single deep image with high $\mathrm{S} / \mathrm{N}$ for each of the four bands separately. Then we applied the GALFIT to decompose the combined images and extract a host galaxy model.

Kim et al. (2017) performed GALFIT on Mrk 590 to model the galaxy and found that the host galaxy has a

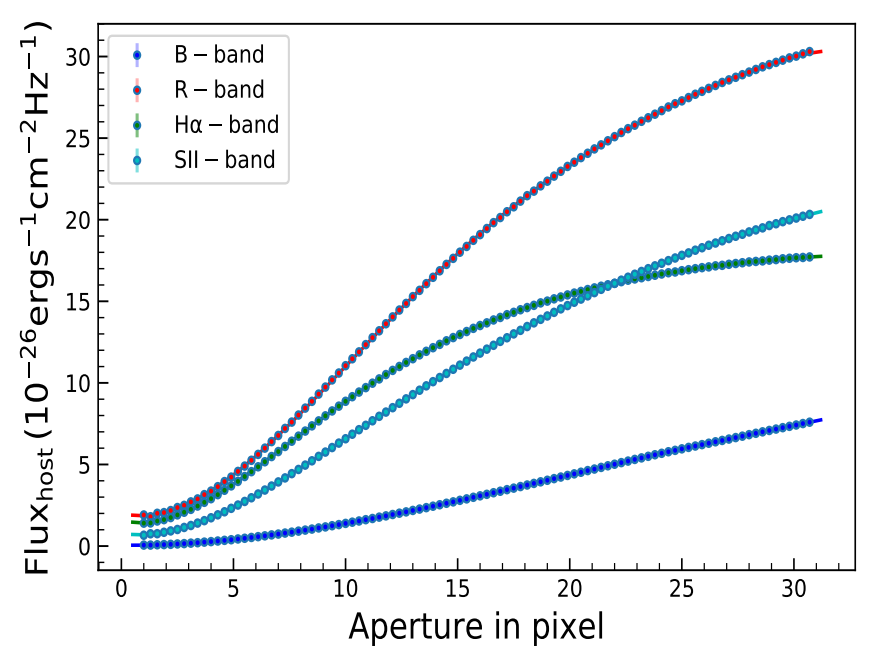

Figure 4. Host-galaxy flux as a function of aperture sizes in $B, R$, $\mathrm{H} \alpha$ and S II bands, respectively. The points with errorbar are the galaxy-flux values and the solid lines are the best polynomial fits. The original $B$-band fluxes are multiplied by 10 for the purpose of presentation.

pseudo bulge with Sérsic index $n=1.04$ and a disk. An exponential disk profile is simply a Sérsic profile with an index of $\mathrm{n}=1$. Following Kim et al. (2017), we used exponential disk profile to model the underlying galaxy of Mrk 590. We generated PSF image from point sources in the combined images. The modeled galaxy image and the residual image that contains the AGN for the S II-band are shown in Fig. 3 (middle) and (right), respectively.

The AGN flux, ideally, can be obtained from the photometry of the residual image that contains only the AGN at the center. But practically, it is very difficult due to poor $\mathrm{S} / \mathrm{N}$ in many epochs of data. Therefore, we carried out aperture photometry on the modeled galaxy image at different concentric aperture sizes and the estimated galaxy-fluxes are plotted against the aperture in Fig. 4. Here, the solid lines show the best-fit curves representing the functional forms of the galaxy light distribution. We performed aperture photometry of the target Mrk 590 at an aperture equal to the aperture radius used for the comparison stars at each individual epoch. We obtained the host-galaxy fluxes from the best fit curve at the aperture used for the photometry of Mrk 590. Then we subtracted the host-galaxy fluxes from the observed total fluxes in those filters to get the host free AGN flux.

\subsection{Flux Calibration}

The instrumental magnitudes obtained from aperture photometry in $B, R, \mathrm{H} \alpha$ and S II bands were converted to fluxes through differential photometry using the following steps:

1. We collected $u$ and $g$-band apparent magnitudes from the SDSS database for a few comparison stars with the best $\mathrm{S} / \mathrm{N}$ present in the same observed frame. The $B$-band apparent magnitudes of those comparison stars were obtained 
from $\operatorname{SDSS} u$ and $g$ - magnitudes using the relation ${ }^{3}$ given below

$B=u-0.8116(u-g)+0.1313$

Then we converted the $B$-band instrumental magnitude to the standard $B$-band magnitude of Mrk 590 using differential photometry of the comparison stars. We collected the $R$-band standard magnitudes of the comparison stars from Zacharias et al. (2017) and the $R$-band magnitude of Mrk 590 was obtained using differential photometry of the comparison stars.

2. Narrow $\mathrm{H} \alpha$ and S II -filters have a mean wavelength of $\sim 6563 \AA$ and $\sim 6719 \AA$ (Andjelić et al. 2011), respectively. The SDSS $r$-band has wavelength coverage from 5380 $\AA$ to $7230 \AA$ with the central wavelength at $6166 \AA$. Therefore, both the narrow $\mathrm{H} \alpha$ and S II band completely fall on the SDSS $r$-band. Hence, we collected the $r$-band apparent magnitudes of the comparison stars from the SDSS database and obtained the standard magnitude of Mrk 590 in both $\mathrm{H} \alpha$ and S II filters using differential photometry of the comparison stars.

The apparent magnitudes were corrected for Galactic extinction taken from the NASA/IPAC Extragalactic data base (NED) ${ }^{4}$ for the broad $B$ and $R$ bands and for the narrow $\mathrm{H} \alpha$ and S II bands, Galactic extinction in r-band was used. The extinction corrected $B$ and $R$-band magnitudes of Mrk 590 were converted to fluxes using the conversion factors given in Bessell et al. (1998). Similarly, to convert the $\mathrm{H} \alpha$ and S II magnitudes of Mrk 590, we used the SDSS r-band conversion factor ${ }^{5}$.

\subsection{Generation of $\mathrm{H} \alpha$ emission line light curve}

The accumulated data on Mrk 590, enables generation of $\mathrm{H} \alpha$ emission line light curve by three methods.

\subsubsection{Method 1}

The observed flux in the narrow S II-band $\left(F_{\mathrm{SII}, \text { tot }}\right)$ contains both $\mathrm{H} \alpha$ line $\left(F_{\mathrm{H} \alpha}\right)$ and continuum fluxes $\left(F_{\text {cont }}\right)$. The variable continuum originates from the accretion disk whose contribution to the observed S II-band fluxes would make the derived lag between the optical/UV continuum and $\mathrm{H} \alpha$ line shorter than the actual lag (Koshida et al. 2014). The accretion disk contributions to the observed line fluxes need to be removed first to get the actual time lag between the $B$ and $\mathrm{H} \alpha$ light curves. The observations in the narrow $\mathrm{H} \alpha$-band contains the continuum flux $\left(F_{\text {cont,H } \alpha}\right)$ from the accretion disk. Hence, $F_{\mathrm{H} \alpha}$ can be obtained from the observed $F_{\mathrm{S} \text { II,tot }}$ as follows

$$
F_{\mathrm{H} \alpha}(t)=F_{\mathrm{S} \text { II,tot }}(t)-F_{\mathrm{cont}, \mathrm{H} \alpha}(t)
$$

3 https://www.sdss.org/dr12/algorithms/sdssUBVRITransform/ \#Lupton2005

4 http://nedwww.ipac.caltech.edu

5 https://www.sdss.org/dr12/algorithms/fluxcal/
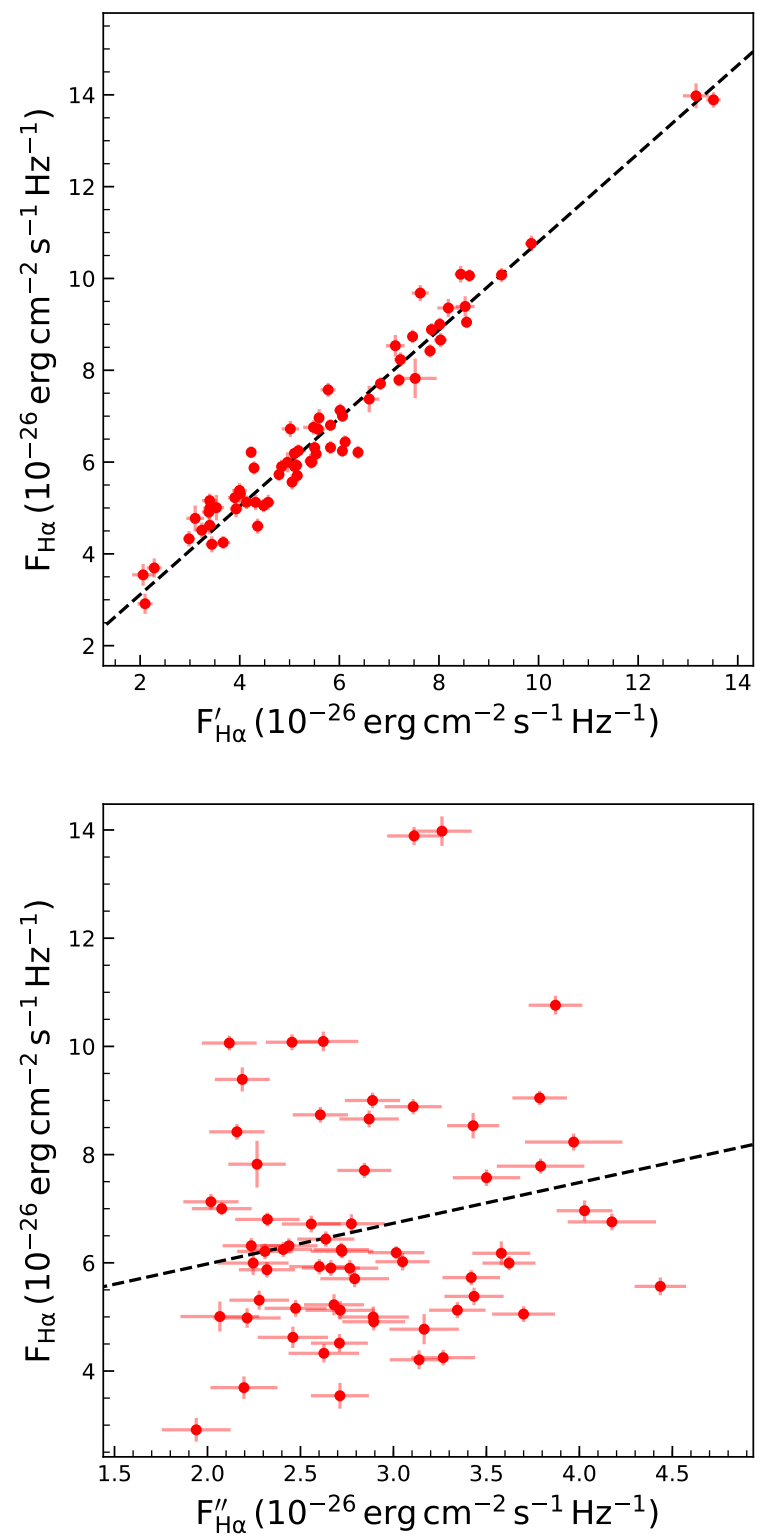

Figure 5. The $\mathrm{H} \alpha$ fluxes $\left(\mathrm{F}_{\mathrm{H} \alpha}\right)$ evaluated using the continuum observations done in the narrow $\mathrm{H} \alpha$ filter as a function of $\mathrm{H} \alpha$ fluxes $\left(\mathrm{F}_{\mathrm{H} \alpha}^{\prime}\right)$ evaluated using broad B-band (top) and that $\left(\mathrm{F}_{\mathrm{H} \alpha}^{\prime \prime}\right)$ obtained from R-band using PL spectrum of accretion disk (bottom). The dashed lines represent the best linear fits. Near simultaneous observations between the narrow $\mathrm{H} \alpha$ filter and the broad $B$ and $R$-bands were used in both the plots.

\subsubsection{Method 2}

The continuum contribution from the accretion disk to the observed S II-band flux can also be estimated from the observed B-band data. This is done by considering a power-law (PL) spectrum of the accretion disk given by Koshida et al. (2014)

$F_{\mathrm{S} \mathrm{II}, \text { cont }}(t)=F_{B}(t)\left(\frac{\nu_{\mathrm{S} \mathrm{II}}}{\nu_{B}}\right)^{\alpha}$

where $F_{\mathrm{SII} \text {,cont }}(\mathrm{t})$ and $F_{B}(\mathrm{t})$ represent the continuum flux of the S II-band from the accretion disk and the $B$-band 
flux at time t, respectively. The $\nu_{B}$ and $\nu_{\mathrm{S} \text { II }}$ are the effective frequencies of the $B$ and S II bands while $\alpha$ is the power-law index. According to Tomita et al. (2006), the power-law index of the accretion-disk component of the flux in the optical and near-infrared may vary from -0.1 to +0.4 . Kishimoto et al. (2008) found $\alpha=0.44 \pm 0.11$ from spectropolarimetric observations. Using the quasar composite spectrum observed by the Sloan Digital Sky Survey, Wilhite et al. (2005) obtained a power-law index of $\alpha=0$. We calculated the continuum contribution in the S II-band flux for $\alpha=1 / 3$ as prescribed by standard accretion disk model (Shakura \& Sunyaev 1973). Finally, we subtracted the $F_{\mathrm{S} I I, c o n t}$ from the observed S II-flux to get the $\mathrm{H} \alpha$ line flux $\left(F_{\mathrm{H} \alpha}^{\prime}\right)$ as

$F_{\mathrm{H} \alpha}^{\prime}(t)=F_{\mathrm{S} \mathrm{II}, \text { tot }}(t)-F_{\mathrm{SII}, \text { cont }}(t)$

The $\mathrm{H} \alpha$ fluxes evaluated using the continuum observations done in the narrow $\mathrm{H} \alpha$ filter (Equation 2) and broad B-band (Equation 4) are found to follow a linear trend, however $F_{\mathrm{H} \alpha}$ is always larger than $F_{\mathrm{H} \alpha}^{\prime}$. This is shown in Fig. 5 (top) that includes those points which have near simultaneous observations in $\mathrm{B}$ and $\mathrm{H} \alpha$ filters. From linear least squares fit to the data, we found

$F_{\mathrm{H} \alpha}=(0.96 \pm 0.03) F_{\mathrm{H} \alpha}^{\prime}+1.19 \pm 0.17$

\subsubsection{Method 3}

The observed $R$-band fluxes contain continuum contribution from the accretion disk and the redshifted $\mathrm{H} \alpha$ line flux from the BLR. To get a lag between $B$ and the $\mathrm{H} \alpha$ line flux derived using the observations in the broad $R$-band, it is necessary to subtract the continuum contribution from the observed $R$-band flux. However, such decomposition is difficult to perform as the AGN continuum is the major contributor to the total flux. Assuming a PL spectrum of accretion disk, we measured the AGN continuum contribution to the observed host-corrected $R$-band fluxes from the near simultaneous $B$ band fluxes as

$F_{\mathrm{R}, \mathrm{cont}}(t)=F_{B}(t)\left(\frac{\nu_{R}}{\nu_{B}}\right)^{\alpha}$

We used $\alpha=1 / 3$ considering a standard accretion disk. From the observed $R$-band fluxes the derived $R$-band continuum was subtracted to get the $\mathrm{H} \alpha$ line fluxes as

$F_{\mathrm{H} \alpha}^{\prime \prime}(t)=F_{R}(t)-F_{\mathrm{R}, \mathrm{cont}}(t)$

The $\mathrm{H} \alpha$ fluxes $\left(\mathrm{F}_{\mathrm{H} \alpha}\right)$ evaluated using the continuum observations done in the narrow $\mathrm{H} \alpha$ filter (Equation 2) are plotted as a function of the $\mathrm{H} \alpha$ line fluxes $\left(\mathrm{F}_{\mathrm{H} \alpha}^{\prime \prime}\right)$ derived from Equation 7 in Fig 5 (bottom). We could not find any strong correlation between $\mathrm{F}_{\mathrm{H} \alpha}^{\prime \prime}$ and $\mathrm{F}_{\mathrm{H} \alpha}$. From linear least squares fit to the data points, we found a Pearson correlation coefficient of $r=0.152$ with a $p$ value of 0.228 . This indicates that it is not possible to derive $\mathrm{H} \alpha$ line fluxes from broad R-band observations. The final light curve data except that of $\mathrm{F}_{\mathrm{H} \alpha}^{\prime \prime}$ are given in Table 2.

\section{ANALYSIS}

\subsection{Light curves}

The light curves of Mrk 590 in the optical $B, R$ and narrow $\mathrm{H} \alpha$ and $\mathrm{S}$ II bands and $\mathrm{H} \alpha$ line generated using the procedures from section 2.6 are shown in Fig. 6. The errors in the flux measurements are obtained through the standard propagation of errors. The top four panels in Fig. 6 show the observed light curves in the broad $B, R$ and narrow $\mathrm{H} \alpha$ and S II filters, while the subsequent three panels show the $\mathrm{H} \alpha$ line light curves derived using Equation 2, 4 and 7 , respectively. The $\mathrm{H} \alpha$ line light curve obtained from the broad $R$-band data is noisy, which indicates the difficulty in getting line fluxes from broad band observations. There is negligible contamination from the radio jet to the observed flux variations as the source is a Seyfert galaxy and considered to be radio-quiet. The characteristics of the light curves are given in Table 3 . To characterise the variability, we used the normalized excess variance $\left(F_{\text {var }}\right.$; Edelson et al. 2002; Vaughan et al. 2003; Rani et al. 2017). The uncertainties in $F_{\text {var }}$ were obtained following Edelson et al. (2002). $R_{\max }$ and $\sigma$ represent the ratio between the maximum and minimum flux and standard deviation of the light curves, respectively. We calculated the host galaxy $\left(L_{\text {host }}\right)$ and AGN luminosity ( $\left.L_{\mathrm{AGN}}\right)$ from the average fluxes obtained for the host galaxy and AGN, respectively, for all the bands and $\mathrm{H} \alpha$ line.

\subsection{Cross-correlation function analysis}

\subsubsection{B-band $v / s$ narrow band light curves}

Mrk 590 shows strong variations in the optical $B$-band and $\mathrm{H} \alpha$ line light curves (see Fig. 6). The $\mathrm{H} \alpha$ light curves used here were derived (a) by subtracting the observed fluxes in the narrow $\mathrm{H} \alpha$ band from that measured in the narrow S II band and (b) by subtracting the continuum obtained by extrapolating the observed B-band flux using $\alpha=1 / 3$ from the measured S II fluxes. To calculate the time lag between the $B$-band and the $\mathrm{H} \alpha$ line light curves, we employed two wellknown methods, namely the interpolated cross-correlation function (ICCF; Gaskell \& Sparke 1986; Gaskell \& Peterson 1987) and the discrete cross-correlation function (DCF; Edelson \& Krolik 1988). The total duration of S II-band data is $\sim 160$ days. Hence, we first evaluated the CCF in the range -50 days to +50 days considering the total duration of observation should exceed at least 3 times the expected maximum lag to locate the peak of the CCF (Peterson \& Horne 2004). The CCFs (Fig. 7) show two peaks; one is at around -6 days and another is at around +22 days. Since a negative lag is physically not possible, we used the centroid of the CCF around the positive peak at +22 days to estimate the expected BLR lag between the $B$-band and the $\mathrm{H} \alpha$ line. The centroid position was estimated by considering the points that are within 80 per cent of the maximum of the CCF as shown in Fig. 8. The cross-correlation centroid distribution (CCCD) obtained using ICCF via Monte Carlo simulation based on the flux randomization and random subset selection described in Peterson et al. (1998a) for 20000 iterations is shown in the histogram in each panel of Fig. 8. The median of the CCCD is taken as a representation of the lag, which is found to be about 22.00 days in the observed frame. Since the distribution has a non-Gaussian 
Table 2. Results of photometry. The fluxes and their uncertainties in different photometric bands are in units of $10^{-26} \mathrm{erg} \mathrm{s}^{-1} \mathrm{~cm}^{-2} \mathrm{~Hz}^{-1}$. MJD in days is given as MJD - 58310.0.

\begin{tabular}{|c|c|c|c|c|c|c|c|c|c|c|c|c|c|c|c|c|c|}
\hline \multicolumn{3}{|c|}{$B$-band } & \multicolumn{3}{|c|}{$R$-band } & \multicolumn{3}{|c|}{$S I I$-band } & \multicolumn{3}{|c|}{$\mathrm{H} \alpha$-band } & \multicolumn{3}{|c|}{$\mathrm{H} \alpha$ line } & & $\mathrm{H} \alpha^{\prime}$ line & \\
\hline MJD & $\mathrm{F}$ & $\sigma$ & MJD & $\mathrm{F}$ & $\sigma$ & MJD & $\mathrm{F}$ & $\sigma$ & MJD & F & $\sigma$ & MJD & $\mathrm{F}$ & $\sigma$ & MJD & F & $\sigma$ \\
\hline 4 & 2.891 & 0.075 & 3 & 4.282 & 0.146 & 10 & 6.624 & 0.121 & 3 & 1.191 & 0.111 & 10 & 5.122 & 0.170 & 10 & 4.318 & 0.164 \\
\hline 5 & 2.554 & 0.045 & 10 & 5.056 & 0.150 & 13 & 7.874 & 0.110 & 10 & 1.502 & 0.120 & 13 & 6.756 & 0.155 & 13 & 5.481 & 0.207 \\
\hline 10 & 2.663 & 0.127 & 13 & 6.606 & 0.158 & 14 & 6.083 & 0.104 & 12 & 1.237 & 0.394 & 14 & 4.604 & 0.165 & 14 & 4.359 & 0.120 \\
\hline 12 & 2.764 & 0.202 & 14 & 4.531 & 0.144 & 18 & 6.281 & 0.109 & 13 & 1.118 & 0.110 & 18 & 5.123 & 0.163 & 18 & 4.571 & 0.127 \\
\hline 26 & 1.919 & 0.109 & 18 & 5.024 & 0.143 & 25 & 6.172 & 0.099 & 14 & 1.479 & 0.128 & 25 & 5.052 & 0.145 & 25 & 4.480 & 0.113 \\
\hline 29 & 1.990 & 0.069 & 25 & 5.388 & 0.139 & 26 & 5.649 & 0.106 & 15 & 0.689 & 0.566 & 26 & 4.247 & 0.144 & 26 & 3.668 & 0.140 \\
\hline 30 & 1.975 & 0.074 & 26 & 4.956 & 0.142 & 28 & 4.198 & 0.205 & 18 & 1.158 & 0.121 & 28 & 3.543 & 0.239 & 28 & 2.060 & 0.220 \\
\hline 31 & 1.955 & 0.062 & 28 & 4.463 & 0.143 & 30 & 15.234 & 0.250 & 19 & 0.615 & 0.159 & 30 & 13.978 & 0.272 & 30 & 13.162 & 0.262 \\
\hline 32 & 2.287 & 0.106 & 29 & 4.024 & 0.144 & 31 & 5.224 & 0.109 & 22 & 1.227 & 0.147 & 31 & 4.515 & 0.168 & 31 & 3.238 & 0.131 \\
\hline 33 & 2.469 & 0.093 & 30 & 4.999 & 0.146 & 32 & 7.204 & 0.103 & 25 & 1.120 & 0.106 & 32 & 5.931 & 0.142 & 32 & 5.139 & 0.119 \\
\hline 34 & 2.393 & 0.093 & 31 & 4.429 & 0.143 & 34 & 6.079 & 0.095 & 26 & 1.402 & 0.098 & 34 & 5.380 & 0.161 & 34 & 3.996 & 0.148 \\
\hline 35 & 2.293 & 0.084 & 32 & 4.614 & 0.132 & 37 & 6.370 & 0.102 & 28 & 0.655 & 0.122 & 37 & 5.125 & 0.149 & 37 & 4.136 & 0.166 \\
\hline 36 & 2.629 & 0.069 & 34 & 5.538 & 0.136 & 39 & 9.815 & 0.133 & 29 & 0.809 & 0.117 & 39 & 9.682 & 0.174 & 39 & 7.625 & 0.168 \\
\hline 37 & 2.384 & 0.068 & 37 & 5.441 & 0.139 & 40 & 7.823 & 0.113 & 30 & 1.256 & 0.109 & 40 & 7.573 & 0.153 & 40 & 5.775 & 0.151 \\
\hline 40 & 2.406 & 0.131 & 40 & 5.617 & 0.140 & 42 & 6.927 & 0.127 & 31 & 0.708 & 0.128 & 42 & 6.722 & 0.176 & 42 & 5.017 & 0.174 \\
\hline 41 & 2.580 & 0.151 & 42 & 4.999 & 0.146 & 43 & 4.886 & 0.161 & 32 & 1.273 & 0.098 & 43 & 4.773 & 0.280 & 43 & 3.105 & 0.177 \\
\hline 42 & 2.529 & 0.119 & 43 & 5.390 & 0.155 & 45 & 5.773 & 0.121 & 34 & 0.699 & 0.129 & 45 & 4.980 & 0.180 & 45 & 3.927 & 0.129 \\
\hline 47 & 2.365 & 0.116 & 45 & 4.293 & 0.149 & 46 & 3.937 & 0.142 & 35 & 0.475 & 0.344 & 46 & 3.693 & 0.208 & 46 & 2.283 & 0.151 \\
\hline 49 & 2.206 & 0.137 & 46 & 4.276 & 0.149 & 47 & 3.655 & 0.142 & 36 & 0.684 & 0.196 & 47 & 2.914 & 0.220 & 47 & 2.099 & 0.151 \\
\hline 55 & 2.056 & 0.085 & 47 & 4.020 & 0.155 & 48 & 5.191 & 0.117 & 37 & 1.245 & 0.109 & 48 & 4.623 & 0.197 & 48 & 3.397 & 0.129 \\
\hline 56 & 2.132 & 0.052 & 48 & 4.400 & 0.146 & 49 & 5.567 & 0.112 & 39 & 0.133 & 0.112 & 49 & 4.998 & 0.194 & 49 & 3.401 & 0.135 \\
\hline 57 & 1.910 & 0.061 & 49 & 4.832 & 0.149 & 50 & 5.094 & 0.101 & 40 & 0.250 & 0.102 & 50 & 4.328 & 0.170 & 50 & 2.982 & 0.117 \\
\hline 58 & 1.797 & 0.057 & 50 & 4.567 & 0.147 & 52 & 5.677 & 0.085 & 41 & 0.485 & 0.130 & 52 & 4.911 & 0.160 & 52 & 3.381 & 0.140 \\
\hline 59 & 2.072 & 0.064 & 52 & 4.703 & 0.151 & 53 & 5.753 & 0.103 & 42 & 0.205 & 0.121 & 53 & 5.160 & 0.154 & 53 & 3.397 & 0.155 \\
\hline 60 & 2.501 & 0.086 & 53 & 4.283 & 0.149 & 54 & 7.768 & 0.120 & 44 & 0.112 & 0.229 & 54 & 6.717 & 0.155 & 54 & 5.572 & 0.137 \\
\hline 62 & 2.439 & 0.067 & 54 & 4.367 & 0.139 & 56 & 7.199 & 0.096 & 45 & 0.792 & 0.133 & 56 & 6.247 & 0.138 & 56 & 5.178 & 0.154 \\
\hline 63 & 2.652 & 0.129 & 55 & 4.548 & 0.138 & 57 & 7.017 & 0.094 & 46 & 0.244 & 0.152 & 57 & 5.901 & 0.153 & 57 & 4.841 & 0.110 \\
\hline 65 & 2.721 & 0.135 & 56 & 4.283 & 0.149 & 58 & 7.354 & 0.085 & 47 & 0.741 & 0.168 & 58 & 6.020 & 0.162 & 58 & 5.422 & 0.121 \\
\hline 68 & 2.536 & 0.077 & 57 & 4.446 & 0.143 & 60 & 8.145 & 0.082 & 48 & 0.569 & 0.159 & 60 & 7.001 & 0.121 & 60 & 6.063 & 0.104 \\
\hline 69 & 2.334 & 0.139 & 58 & 4.630 & 0.137 & 61 & 9.933 & 0.119 & 52 & 0.766 & 0.136 & 61 & 8.659 & 0.157 & 61 & 8.032 & 0.133 \\
\hline 72 & 2.513 & 0.068 & 60 & 4.276 & 0.142 & 62 & 9.577 & 0.416 & 53 & 0.594 & 0.115 & 62 & 7.823 & 0.432 & 62 & 7.523 & 0.431 \\
\hline 73 & 2.231 & 0.099 & 61 & 5.069 & 0.141 & 63 & 9.089 & 0.101 & 54 & 1.051 & 0.099 & 63 & 7.787 & 0.139 & 63 & 7.198 & 0.108 \\
\hline 74 & 2.404 & 0.074 & 62 & 4.412 & 0.143 & 65 & 10.365 & 0.118 & 55 & 0.909 & 0.101 & 65 & 10.092 & 0.181 & 65 & 8.437 & 0.123 \\
\hline 75 & 2.195 & 0.068 & 63 & 6.124 & 0.206 & 66 & 10.264 & 0.168 & 56 & 0.952 & 0.099 & 66 & 9.357 & 0.199 & 66 & 8.188 & 0.221 \\
\hline 81 & 2.373 & 0.130 & 65 & 5.016 & 0.146 & 68 & 7.439 & 0.106 & 57 & 1.116 & 0.121 & 68 & 6.314 & 0.140 & 68 & 5.503 & 0.114 \\
\hline 83 & 2.184 & 0.045 & 68 & 4.668 & 0.139 & 69 & 7.062 & 0.104 & 58 & 1.334 & 0.138 & 69 & 5.706 & 0.157 & 69 & 5.154 & 0.110 \\
\hline 84 & 2.226 & 0.042 & 69 & 4.844 & 0.138 & 70 & 6.726 & 0.113 & 59 & 1.435 & 0.102 & 70 & 5.995 & 0.218 & 70 & 4.960 & 0.141 \\
\hline 85 & 2.397 & 0.165 & 70 & 4.299 & 0.146 & 73 & 6.690 & 0.076 & 60 & 1.144 & 0.090 & 73 & 6.213 & 0.129 & 73 & 4.229 & 0.088 \\
\hline 86 & 2.235 & 0.048 & 73 & 4.686 & 0.144 & 74 & 6.672 & 0.084 & 61 & 1.274 & 0.103 & 74 & 5.872 & 0.142 & 74 & 4.286 & 0.093 \\
\hline 87 & 2.203 & 0.041 & 74 & 4.434 & 0.136 & 75 & 6.238 & 0.088 & 62 & 1.755 & 0.115 & 75 & 5.310 & 0.177 & 75 & 4.013 & 0.099 \\
\hline 91 & 2.039 & 0.099 & 75 & 4.209 & 0.149 & 80 & 5.541 & 0.150 & 63 & 1.303 & 0.095 & 80 & 5.007 & 0.278 & 80 & 3.532 & 0.153 \\
\hline 93 & 2.842 & 0.051 & 80 & 4.154 & 0.178 & 83 & 8.067 & 0.088 & 65 & 0.273 & 0.138 & 83 & 6.802 & 0.126 & 83 & 5.822 & 0.095 \\
\hline 94 & 2.756 & 0.044 & 83 & 4.243 & 0.169 & 84 & 9.904 & 0.102 & 66 & 0.907 & 0.106 & 84 & 8.735 & 0.145 & 84 & 7.471 & 0.108 \\
\hline 95 & 2.569 & 0.053 & 84 & 4.565 & 0.144 & 85 & 8.939 & 0.199 & 68 & 1.124 & 0.092 & 85 & 7.373 & 0.290 & 85 & 6.600 & 0.203 \\
\hline 96 & 2.320 & 0.035 & 86 & 4.981 & 0.146 & 86 & 7.210 & 0.075 & 69 & 1.356 & 0.118 & 86 & 6.188 & 0.119 & 86 & 5.097 & 0.079 \\
\hline 97 & 2.593 & 0.041 & 87 & 3.956 & 0.144 & 87 & 8.088 & 0.109 & 70 & 0.730 & 0.186 & 87 & 7.128 & 0.143 & 87 & 6.016 & 0.114 \\
\hline 98 & 2.810 & 0.042 & 88 & 5.367 & 0.136 & 88 & 9.279 & 0.185 & 72 & 0.169 & 0.136 & 88 & 8.535 & 0.235 & 88 & 7.124 & 0.188 \\
\hline 99 & 2.702 & 0.045 & 91 & 5.763 & 0.247 & 91 & 9.353 & 0.119 & 73 & 0.477 & 0.104 & 91 & 8.232 & 0.157 & 91 & 7.227 & 0.125 \\
\hline 100 & 2.441 & 0.029 & 93 & 4.617 & 0.140 & 93 & 10.669 & 0.100 & 74 & 0.800 & 0.115 & 93 & 10.062 & 0.137 & 93 & 8.613 & 0.108 \\
\hline 101 & 2.393 & 0.038 & 94 & 4.582 & 0.144 & 94 & 9.811 & 0.096 & 75 & 0.929 & 0.153 & 94 & 8.421 & 0.144 & 94 & 7.821 & 0.115 \\
\hline 102 & 2.489 & 0.039 & 96 & 4.929 & 0.146 & 96 & 10.013 & 0.112 & 80 & 0.534 & 0.234 & 96 & 9.001 & 0.145 & 96 & 8.017 & 0.120 \\
\hline 103 & 2.456 & 0.041 & 97 & 4.735 & 0.136 & 97 & 10.859 & 0.107 & 83 & 1.265 & 0.090 & 97 & 10.077 & 0.148 & 97 & 9.258 & 0.110 \\
\hline 104 & 2.374 & 0.046 & 98 & 5.315 & 0.141 & 98 & 8.834 & 0.095 & 84 & 1.169 & 0.104 & 98 & 7.708 & 0.139 & 98 & 6.825 & 0.101 \\
\hline 106 & 2.298 & 0.074 & 99 & 4.686 & 0.144 & 99 & 8.077 & 0.104 & 85 & 1.567 & 0.211 & 99 & 6.211 & 0.142 & 99 & 6.375 & 0.106 \\
\hline 107 & 2.304 & 0.052 & 100 & 4.783 & 0.152 & 100 & 7.780 & 0.108 & 86 & 1.022 & 0.093 & 100 & 6.439 & 0.142 & 100 & 6.116 & 0.110 \\
\hline 109 & 1.849 & 0.027 & 101 & 4.824 & 0.145 & 101 & 7.877 & 0.087 & 87 & 0.960 & 0.093 & 101 & 6.244 & 0.131 & 101 & 6.061 & 0.090 \\
\hline 112 & 2.320 & 0.041 & 102 & 4.424 & 0.150 & 102 & 7.579 & 0.085 & 88 & 0.744 & 0.145 & 102 & 6.316 & 0.142 & 102 & 5.823 & 0.089 \\
\hline 113 & 1.965 & 0.027 & 103 & 4.824 & 0.152 & 103 & 7.372 & 0.084 & 91 & 1.122 & 0.102 & 103 & 5.903 & 0.145 & 103 & 5.101 & 0.090 \\
\hline 114 & 1.921 & 0.026 & 104 & 6.524 & 0.132 & 104 & 7.011 & 0.101 & 93 & 0.607 & 0.093 & 104 & 5.565 & 0.165 & 104 & 5.051 & 0.104 \\
\hline 115 & 2.097 & 0.029 & 107 & 5.275 & 0.152 & 108 & 7.377 & 0.093 & 94 & 1.390 & 0.107 & 108 & 6.176 & 0.220 & 108 & 5.534 & 0.097 \\
\hline 116 & 2.029 & 0.030 & 108 & 5.607 & 0.149 & 114 & 10.192 & 0.094 & 96 & 1.012 & 0.093 & 114 & 9.047 & 0.131 & 114 & 8.554 & 0.099 \\
\hline 117 & 2.623 & 0.036 & 109 & 5.336 & 0.144 & 115 & 15.155 & 0.141 & 97 & 0.782 & 0.102 & 115 & 13.890 & 0.168 & 115 & 13.512 & 0.144 \\
\hline 118 & 2.263 & 0.031 & 114 & 5.476 & 0.145 & 118 & 10.134 & 0.190 & 98 & 1.127 & 0.102 & 118 & 9.390 & 0.222 & 118 & 8.527 & 0.191 \\
\hline 119 & 2.060 & 0.028 & 115 & 4.956 & 0.142 & 134 & 7.277 & 0.078 & 99 & 1.866 & 0.097 & 134 & 6.963 & 0.191 & 134 & 5.592 & 0.081 \\
\hline 120 & 1.947 & 0.027 & 118 & 4.178 & 0.145 & 135 & 5.629 & 0.140 & 100 & 1.341 & 0.093 & 135 & 5.224 & 0.195 & 135 & 3.902 & 0.142 \\
\hline 121 & 2.073 & 0.029 & 134 & 5.900 & 0.147 & 136 & 5.200 & 0.127 & 101 & 1.634 & 0.098 & 136 & 4.208 & 0.175 & 136 & 3.440 & 0.129 \\
\hline 122 & 2.064 & 0.028 & 135 & 4.344 & 0.158 & 137 & 6.475 & 0.078 & 102 & 1.264 & 0.113 & 137 & 5.728 & 0.138 & 137 & 4.788 & 0.081 \\
\hline 123 & 2.147 & 0.034 & 136 & 4.806 & 0.156 & 138 & 7.119 & 0.075 & 103 & 1.469 & 0.119 & 138 & 5.995 & 0.131 & 138 & 5.443 & 0.079 \\
\hline
\end{tabular}


Table 2 - continued

\begin{tabular}{|c|c|c|c|c|c|c|c|c|c|c|c|c|c|c|c|c|c|}
\hline \multicolumn{3}{|c|}{$B$-band } & \multicolumn{3}{|c|}{$R$-band } & \multicolumn{3}{|c|}{$S I I$-band } & \multicolumn{3}{|c|}{$\mathrm{H} \alpha$-band } & \multicolumn{3}{|c|}{$\mathrm{H} \alpha$ line } & \multicolumn{3}{|c|}{$\mathrm{H} \alpha^{\prime}$ line } \\
\hline MJD & $\mathrm{F}$ & $\sigma$ & MJD & F & $\sigma$ & MJD & F & $\sigma$ & MJD & F & $\sigma$ & MJD & F & $\sigma$ & MJD & F & $\sigma$ \\
\hline 124 & 2.578 & 0.041 & 137 & 5.051 & 0.153 & 140 & 11.560 & 0.127 & 104 & 1.446 & 0.130 & 140 & 10.761 & 0.173 & 140 & 9.855 & 0.129 \\
\hline 125 & 2.736 & 0.046 & 138 & 5.332 & 0.141 & 143 & 9.918 & 0.089 & 107 & 1.419 & 0.130 & 143 & 8.886 & 0.141 & 143 & 7.850 & 0.094 \\
\hline 126 & 3.430 & 0.076 & 140 & 5.659 & 0.142 & 148 & 12.372 & 0.115 & 108 & 1.200 & 0.199 & - & - & - & 148 & 9.862 & 0.121 \\
\hline 127 & 1.853 & 0.029 & 141 & 5.582 & 0.145 & 149 & 11.212 & 0.114 & 114 & 1.145 & 0.092 & - & - & - & 149 & 9.2 & 0.118 \\
\hline 128 & 1.814 & 0.028 & 142 & 4.859 & 0.145 & 152 & 8.924 & 0.089 & 115 & 1.265 & 0.090 & - & - & - & 152 & 6.968 & 0.092 \\
\hline 129 & 2.007 & 0.034 & 143 & 4.819 & 0.152 & 161 & 7.192 & 0.096 & 118 & 0.744 & 0.116 & - & - & - & 161 & 5.05 & 0.1 \\
\hline 130 & 1.773 & 0.029 & 148 & 4.507 & 0.142 & 162 & 7.353 & 0.115 & 119 & 1.184 & 0.199 & - & - & - & 162 & 5.128 & 0.121 \\
\hline 131 & 1.818 & 0.030 & 149 & 4.842 & 0.149 & 163 & 4.197 & 0.161 & 130 & 0.627 & 0.185 & - & - & - & 163 & 2.098 & 0.164 \\
\hline 132 & 1.993 & 0.039 & 150 & 6.303 & 0.160 & 164 & 7.345 & 0.099 & 132 & 0.778 & 0.123 & - & - & - & 164 & 5.336 & 0.105 \\
\hline 134 & 2.128 & 0.034 & 151 & 5.241 & 0.154 & 165 & 7.669 & 0.101 & 134 & 0.314 & 0.174 & - & - & - & 165 & 5.628 & 0.107 \\
\hline 135 & 1.891 & 0.037 & 152 & 5.352 & 0.148 & 166 & 8.542 & 0.089 & 135 & 0.404 & 0.135 & - & - & - & 166 & 6.752 & 0.094 \\
\hline 136 & 1.897 & 0.031 & 153 & 5.214 & 0.144 & 167 & 9.551 & 0.091 & 136 & 0.991 & 0.120 & - & - & - & 167 & 7.738 & 0.094 \\
\hline 137 & 1.856 & 0.027 & 161 & 5.056 & 0.141 & 168 & 10.889 & 0.101 & 137 & 0.747 & 0.114 & - & - & - & 168 & 9.175 & 0.103 \\
\hline 138 & 1.945 & 0.027 & 162 & 5.301 & 0.181 & 169 & 11.479 & 0.091 & 138 & 1.124 & 0.107 & - & - & - & 169 & 9.767 & 0.094 \\
\hline 139 & 1.993 & 0.027 & 163 & 6.693 & 0.148 & 170 & 8.754 & 0.091 & 140 & 0.798 & 0.117 & - & - & - & 170 & 6.848 & 0.095 \\
\hline 140 & 2.032 & 0.028 & 164 & 5.353 & 0.151 & - & - & - & 141 & 1.216 & 0.106 & - & - & - & - & - & - \\
\hline 141 & 1.690 & 0.023 & 165 & 4.774 & 0.175 & - & - & - & 142 & 1.227 & 0.092 & - & - & - & - & - & - \\
\hline 142 & 1.877 & 0.026 & 167 & 5.670 & 0.140 & - & - & - & 143 & 1.033 & 0.109 & - & - & - & - & - & - \\
\hline 143 & 1.948 & 0.026 & 168 & 4.985 & 0.149 & - & - & - & - & - & - & - & - & - & - & - & - \\
\hline 144 & 1.936 & 0.028 & 169 & 5.781 & 0.143 & - & - & - & - & - & - & - & - & - & - & - & - \\
\hline 145 & 1.969 & 0.025 & 170 & 4.531 & 0.150 & - & - & - & - & - & - & - & - & - & - & - & - \\
\hline 146 & 2.389 & 0.033 & 182 & 6.546 & 0.151 & - & - & - & - & - & - & - & - & - & - & - & - \\
\hline 147 & 2.898 & 0.043 & - & - & - & - & - & - & - & - & - & - & - & - & - & - & - \\
\hline 148 & 2.324 & 0.035 & - & - & - & - & - & - & - & - & - & - & - & - & - & - & - \\
\hline 149 & 2.259 & 0.029 & - & - & - & - & - & - & - & - & - & - & - & - & - & - & - \\
\hline 150 & 2.527 & 0.037 & - & - & - & - & - & - & - & - & - & - & - & - & - & - & - \\
\hline 151 & 2.469 & 0.029 & - & - & - & - & - & - & - & - & - & - & - & - & - & - & - \\
\hline 152 & 2.473 & 0.032 & - & - & - & - & - & - & - & - & - & - & - & - & - & - & - \\
\hline 153 & 2.576 & 0.031 & - & - & - & - & - & - & - & - & - & - & - & - & - & - & - \\
\hline 154 & 2.170 & 0.030 & - & - & - & - & - & - & - & - & - & - & - & - & - & - & - \\
\hline 155 & 2.292 & 0.031 & - & - & - & - & - & - & - & - & - & - & - & - & - & - & - \\
\hline 156 & 2.447 & 0.031 & - & - & - & - & - & - & - & - & - & - & - & - & - & - & - \\
\hline 157 & 2.111 & 0.027 & - & - & - & - & - & - & - & - & - & - & - & - & - & - & - \\
\hline 158 & 2.021 & 0.026 & - & - & - & - & - & - & - & - & - & - & - & - & - & - & - \\
\hline 159 & 2.136 & 0.029 & - & - & - & - & - & - & - & - & - & - & - & - & - & - & - \\
\hline 161 & 2.570 & 0.045 & - & - & - & - & - & - & - & - & - & - & - & - & - & - & - \\
\hline 162 & 2.424 & 0.036 & - & - & - & - & - & - & - & - & - & - & - & - & - & - & - \\
\hline 163 & 2.320 & 0.040 & - & - & - & - & - & - & - & - & - & - & - & - & - & - & - \\
\hline 164 & 2.358 & 0.041 & - & - & - & - & - & - & - & - & - & - & - & - & - & - & - \\
\hline 165 & 2.066 & 0.034 & - & - & - & - & - & - & - & - & - & - & - & - & - & - & - \\
\hline 167 & 2.094 & 0.026 & - & - & - & - & - & - & - & - & - & - & - & - & - & - & - \\
\hline 168 & 1.980 & 0.027 & - & - & - & - & - & - & - & - & - & - & - & - & - & - & - \\
\hline 169 & 1.977 & 0.025 & - & - & - & - & - & - & - & - & - & - & - & - & - & - & - \\
\hline 170 & 2.201 & 0.030 & - & - & - & - & - & - & - & - & - & - & - & - & - & - & - \\
\hline 171 & 2.164 & 0.027 & - & - & - & - & - & - & - & - & - & - & - & - & - & - & - \\
\hline 172 & 1.974 & 0.025 & - & - & - & - & - & - & - & - & - & - & - & - & - & - & - \\
\hline 173 & 1.828 & 0.025 & - & - & - & - & - & - & - & - & - & - & - & - & - & - & - \\
\hline
\end{tabular}

Table 3. Variability statistics in $B, R$, narrow $\mathrm{H} \alpha$ and S II bands and for $H_{\alpha}$ line in observer's frame, where $\lambda_{\text {eff }}$ is the effective wavelength in Angstroms. The average values $(\langle f\rangle)$ and the standard deviation $\sigma$ of the light curves are in units of $10^{-26} \mathrm{erg} \mathrm{s}^{-1} \mathrm{~cm}^{-2} \mathrm{~Hz}^{-1}$. The luminosities in each band are in $\operatorname{erg~s}^{-1}$.

\begin{tabular}{lccccccc}
\hline Filter & $\lambda_{\text {eff }}$ & $\langle f\rangle$ & $\sigma$ & $F_{\text {var }}$ & $R_{\max }$ & $\log L_{\text {host }}$ & $\log L_{\text {AGN }}$ \\
\hline$B$ & 4363 & $2.26 \pm 0.05$ & 0.31 & $0.132 \pm 0.001$ & 2.03 & $42.22 \pm 0.01$ & $43.36 \pm 0.01$ \\
$R$ & 6410 & $4.95 \pm 0.15$ & 0.62 & $0.121 \pm 0.001$ & 1.69 & $43.98 \pm 0.01$ & $43.53 \pm 0.01$ \\
$\mathrm{H} \alpha$ & 6563 & $0.97 \pm 0.13$ & 0.40 & $0.379 \pm 0.005$ & 16.60 & $43.85 \pm 0.01$ & $42.81 \pm 0.06$ \\
$\mathrm{~S} \mathrm{II}$ & 6719 & $7.88 \pm 0.12$ & 2.26 & $0.286 \pm 0.001$ & 4.17 & $43.70 \pm 0.01$ & $43.71 \pm 0.01$ \\
$\mathrm{H} \alpha$ line & 6564.6 & $6.73 \pm 0.17$ & 2.17 & $0.321 \pm 0.001$ & 4.80 & - & $43.65 \pm 0.01$ \\
$\mathrm{H} \alpha^{\prime}$ line & 6564.6 & $5.90 \pm 0.13$ & 2.28 & $0.385 \pm 0.001$ & 6.56 & - & $43.60 \pm 0.01$ \\
$\mathrm{H} \alpha^{\prime \prime}$ line & 6564.6 & $2.94 \pm 0.16$ & 0.63 & $0.206 \pm 0.001$ & 2.68 & - & $43.29 \pm 0.02$ \\
\hline
\end{tabular}




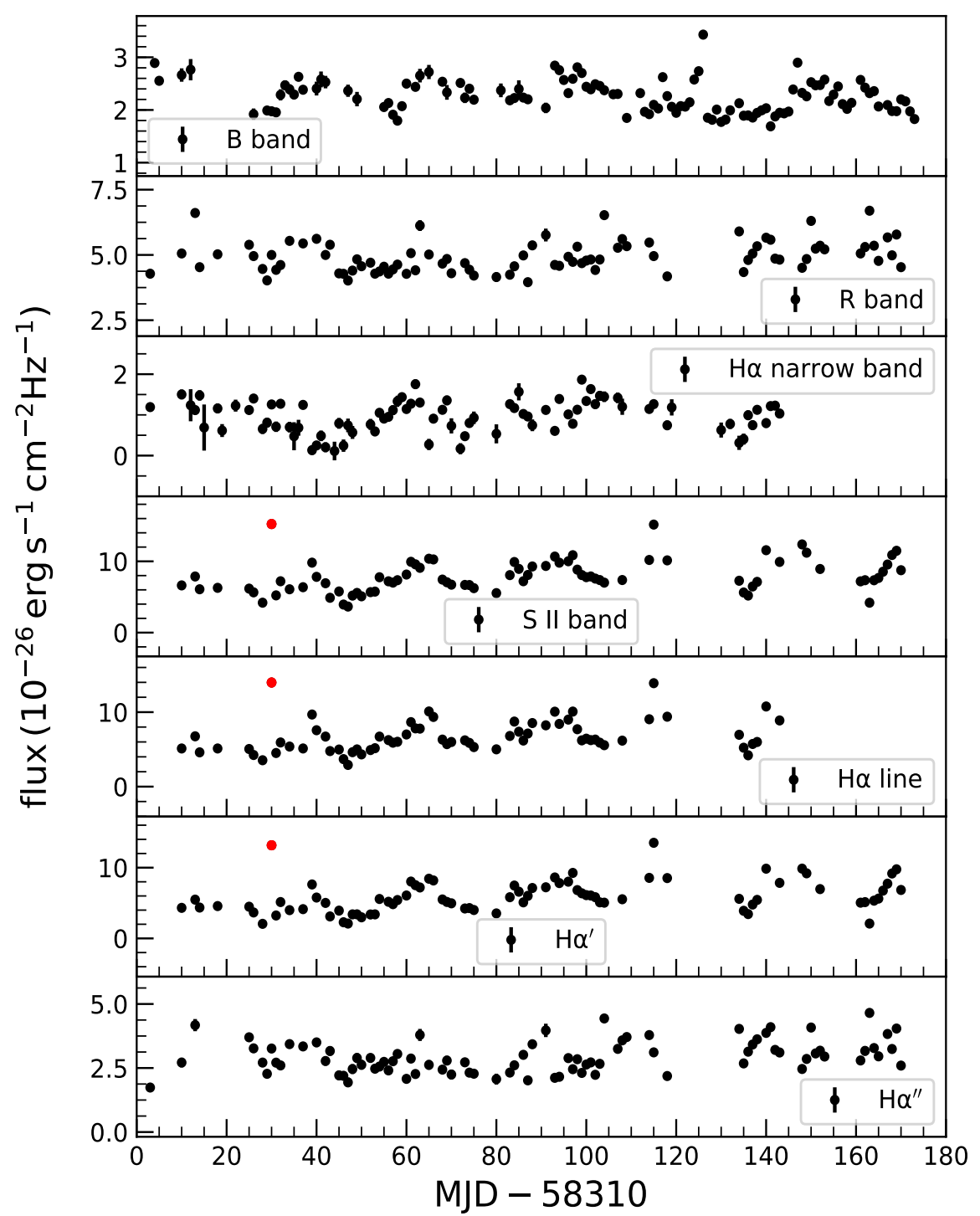

Figure 6. The light curves of Mrk 590 in the broad B- and R-band filters and narrow $\mathrm{H} \alpha-$ and S II band filters for the period from July, 2018 to December, 2018. The flux in the narrow $\mathrm{H} \alpha$-band was subtracted from the S II-band fluxes to get the $\mathrm{H} \alpha$ line light curve, whereas $\mathrm{H} \alpha^{\prime}$ and $\mathrm{H} \alpha^{\prime \prime}$ light curves were obtained considering a PL spectrum of the accretion disk and subtracted from the narrow S II and broad R-band, respectively. The outlier is shown by red circle.

shape, we calculated the uncertainties within $68 \%$ confidence interval around the median value as described in Mandal et al. (2018).

The measured lags and their uncertainties between the $B$-band and $\mathrm{H} \alpha$ line light curves in the observed frame derived from the Monte Carlo simulation using the ICCF analysis method are given in Table 4 . We found similar time lags between the $B$-band continuum and the $\mathrm{H} \alpha$ light curves derived from two approaches. Considering the $\mathrm{H} \alpha$ line light curve derived using Equation 2, which is straight forward devoid of any uncertainties due to the use of a single value of $\alpha$ as in Equations 4 and 7, we found the rest frame time lag between the $B$-band and the $\mathrm{H} \alpha$ line as $21.44_{-2.11}^{+1.49}$ days, which corresponds to a BLR size of $0.018 \mathrm{pc}$. We noticed one outlier point (shown as red circle) in the S II, $\mathrm{H} \alpha$ and $\mathrm{H} \alpha^{\prime}$ light curves in Fig. 6. On examining the S II band image acquired at that epoch, we found the target source to be af- fected by a weak cosmic ray hit. After excluding this outlier point in the S II, $\mathrm{H} \alpha$, and $\mathrm{H} \alpha^{\prime}$ light curves, we carried out the CCF analysis. We found a rest-frame lag of $21.42_{-2.38}^{+1.03}$ days, which is similar to that obtained with the same outlier point included. The impact of this sole outlier point is therefore, minimal in our measured lag.

\subsection{2 $B$-band $v / s$-band light curves}

The CCF between the $B$ and $R$-band light curves is shown in Fig. 9 (top). The host corrected $R$-band has contributions from the continuum as well as the $\mathrm{H} \alpha$ line fluxes (see Fig. 1). This is also reflected in the CCF (Fig. 9; top panel), which shows two prominent peaks; one at a smaller lag of $\sim 3$ days due to the contribution from the continuum flux originating from the accretion disk, and another at lag of $\sim 16$ days, which is due to the contribution from the $\mathrm{H} \alpha$ line flux 


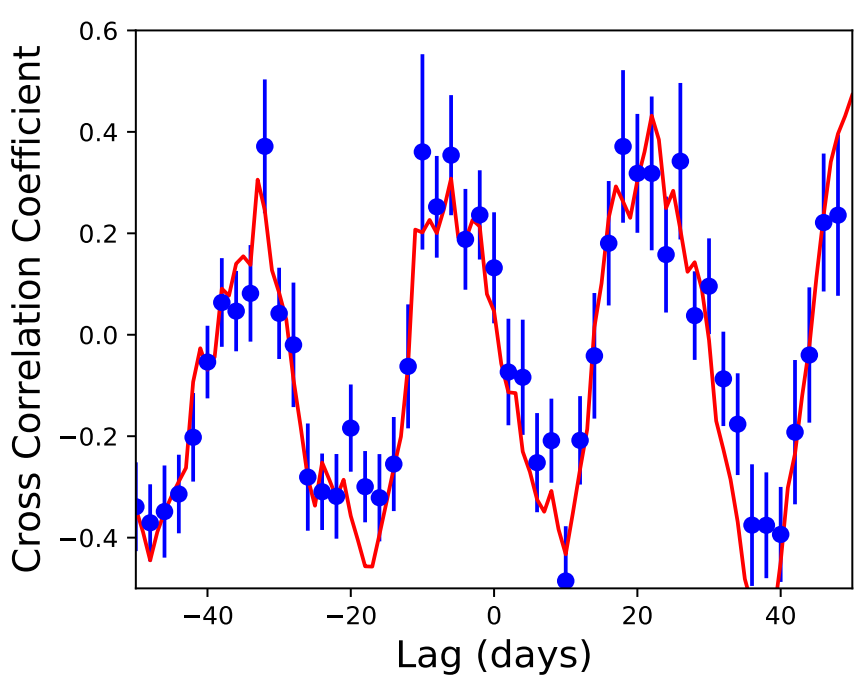

Figure 7. The CCFs between $\mathrm{B}$ and $\mathrm{H} \alpha$ light curves. The solid red line represents the ICCF, points with error bars show the DCF obtained using $\Delta \tau=1$ day.

from the BLR. Recent disk reverberation mapping observations utilizing high quality data on Fairall 9 and Mrk 110 by Hernández Santisteban et al. (2020) and Vincentelli et al. (2021), respectively, show well correlated variations between different optical bands. However, we note a weak correlation between the continuum B and R-band light curves with a peak of CCF of $\mathrm{r}_{\max } \sim 0.1$. Such a low correlation is due to a combination of the following factors (a) our light curves are of moderate quality, (b) the B-band light curve is dominated by the AGN, while the R-band light curve is contaminated to a large extent by light from the host galaxy. Though we carried out GALFIT to remove the contribution of the host galaxy to the observed R-band brightness, it was not effective as the seeing was not stable during the observing period. As pointed by Cellone et al. (2000) seeing fluctuations during the observations can severely affect the observed light curves. According to them, degradation in the light curves due to seeing fluctuations are larger for AGN with brighter host. In our case too, (i) compared to B-band, R-band has larger host contamination and (ii) seeing was not stable and has varied between 1.7 to 4.4 arcsec during the monitoring period. Such seeing variations coupled with the larger host galaxy contribution to the R-band measurements have led to poor quality R-band light curve. The B-band light curve when cross-correlated with the poor quality R-band light curve has led to the weak correlation. The R-band light curve having little resemblance to the B-band light curve is also evident in Fig. 6 and (c) the R-band contains contributions from two spatially different regions, namely, the continuum coming from the accretion disk and the redshifted $\mathrm{H} \alpha$ from the BLR

To have only the $\mathrm{H} \alpha$ line contributing to the measured flux in the $R$-band, we subtracted the contribution of the continuum to the observed $R$-band flux using Equation 7 . We then, cross-correlated the $B$-band and continuum subtracted $R$-band ( $\left.\mathrm{H} \alpha^{\prime \prime}\right)$ light curves. We found a lag of $\sim 16$ days in the observed frame with an improved correlation of $\mathrm{r}_{\max } \sim 0.27$ (Fig. 9; bottom panel). Such a weak correlation
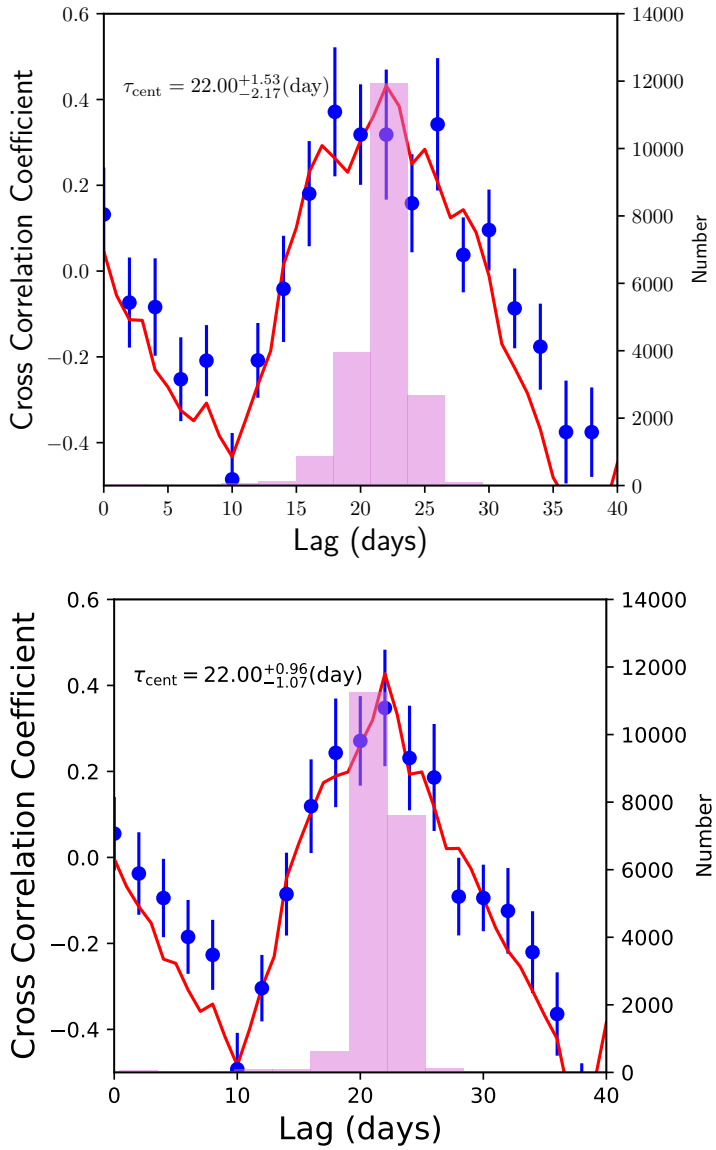

Figure 8. The CCFs between $B$ and $\mathrm{H} \alpha$ line flux (top panel) and the same with $\alpha=1 / 3$ (bottom panel). The solid red line represents the ICCF, points with error bars show the DCF obtained using $\Delta \tau=1$ day and the corresponding distribution of the $\tau_{\text {cent }}$ obtained using 20000 Monte Carlo simulations is shown by histogram in each panel. The value of $\tau_{c e n t}$ from the median of the distribution obtained using Monte Carlo simulation using ICCF is noted in each panel.

has also been found in other studies where reliable lag measurements have been claimed, e.g., broad band photometric RM has been carried out for NGC 4395 by Edri et al. (2012) between SDSS $g, r$ and $i$ filters, which provided a BLR size but with a weak correlation of $r_{\max } \sim 0.1$. The measured lag with uncertainties between $B$ and continuum subtracted $R$-band is given in Table 4. The lag between $B$ and $\mathrm{H} \alpha$ line light curve obtained from continuum subtracted narrow S II band data is slightly larger compared to that of using continuum subtracted $R$-band as $\mathrm{H} \alpha$ line flux. This is because the narrow S II band has much smaller contribution from AGN continuum than in broad $R$-band (Koshida et al. 2014).

Photometric continuum RM observations (Fausnaugh et al. 2016, 2018; Homayouni et al. 2021) suggest that continuum lags $(\tau)$ increase with wavelength $(\lambda)$ with $\tau \propto \lambda^{4 / 3}$ as expected from the standard accretion disk model by Shakura \& Sunyaev (1973). Following Homayouni et al. (2021), the accretion disk size can be parameterized as:

$\tau_{\text {optical }}-\tau_{u v}=\tau_{0}\left[\left(\frac{\lambda_{\text {optical }}}{2700 \AA}\right)^{\beta}-\left(\frac{\lambda_{u v}}{2700 \AA}\right)^{\beta}\right]$ 

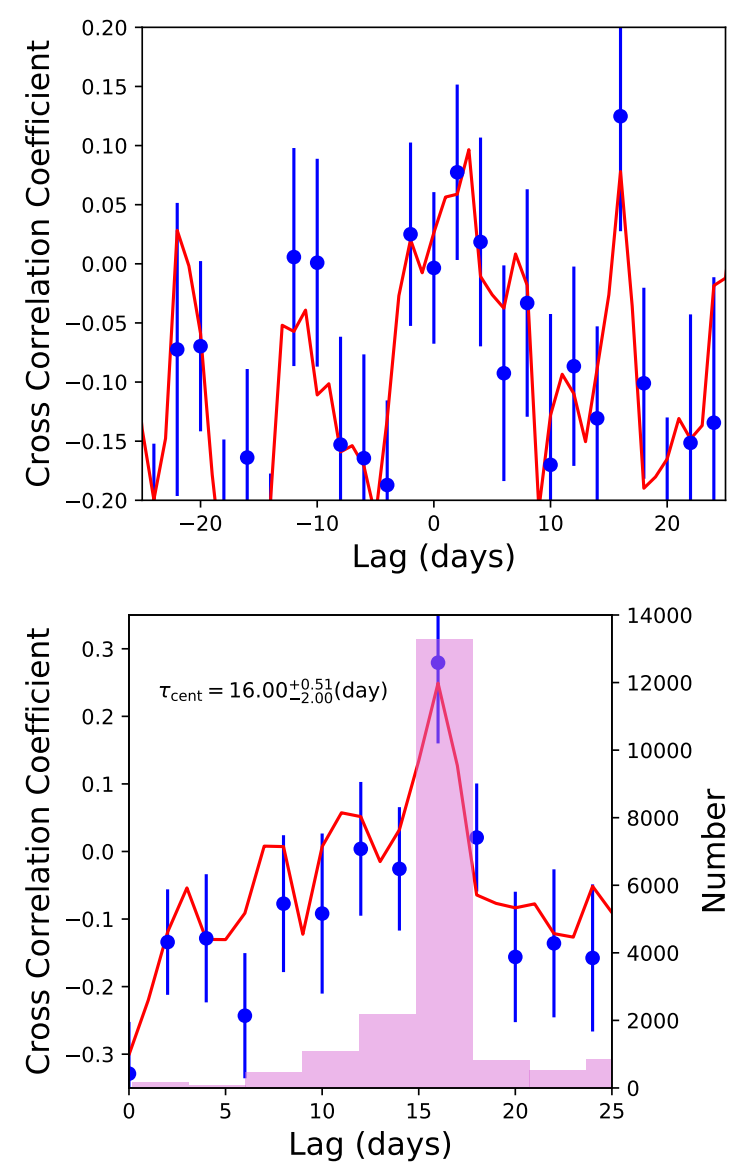

Figure 9. The CCFs between $B$ and $R$ (top panel) and continuum subtracted $R\left(\mathrm{H} \alpha^{\prime \prime}\right)$ with $\alpha=1 / 3$ (bottom panel) are shown. The solid red line represents the ICCF, points with error bars show the DCF obtained using $\Delta \tau=1$ day and the corresponding distribution of the $\tau_{\text {cent }}$ obtained using 20000 Monte Carlo Simulations is shown by histogram. The value of $\tau_{\text {cent }}$ from the median of the distribution obtained using Monte Carlo simulation using ICCF is also noted in the bottom panel.

where, $\tau$ represents the rest frame lag, $\lambda_{\mathrm{uv}}$ and $\lambda_{\text {optical }}$ are the rest frame UV and optical reference wavelengths, respectively, with $\beta=4 / 3$ for a standard accretion disk (Shakura \& Sunyaev 1973). Therefore, using Equation 8, the lag between B and R-band continua can be obtained as:

$\tau_{R}-\tau_{B}=\tau_{0}\left[\left(\frac{\lambda_{R}}{2700 \AA}\right)^{\beta}-\left(\frac{\lambda_{B}}{2700 \AA}\right)^{\beta}\right]$

with the disk normalization parameter $\tau_{0}$ given as

$$
\begin{array}{r}
\tau_{0}=\frac{1}{c}\left(\frac{45 G}{16 \pi^{6} h c^{2}}\right)^{1 / 3}(2700 \AA)^{4 / 3} \chi^{4 / 3}\left(\frac{A_{b o l}}{\eta c^{2}}\right)^{1 / 3} \\
\left\langle M_{B H}\right\rangle^{1 / 3}\left\langle\lambda L_{\lambda}\right\rangle^{1 / 3}
\end{array}
$$

Here, we assumed the geometrical factor related to the flux-weighted mean radius, $\chi=2.49$ as suggested by Homayouni et al. (2021), radiative efficiency $\eta=0.1$, bolometric luminosity $\mathrm{L}_{\mathrm{bol}}=\mathrm{A}_{\mathrm{bol}} \lambda \mathrm{L}_{\lambda}$, where $\lambda L_{\lambda}$ was obtained from the monochromatic luminosity at $5100 \AA$ with $\mathrm{A}_{\text {bol }}=$ 8.1 \pm 0.4 (Runnoe et al. 2012). Using Equations 9 and 10, we found the expected lag between B and R-band continua as
$0.32_{-0.01}^{+0.01}$ days, where the uncertainties were obtained using the propagation of error method. The expected continuum lag from the continuum-lag- wavelength relation is found to be very small compared to the BLR size obtained based on lag between B-band and continuum subtracted $\mathrm{H} \alpha$ line light curves. This implies that the inherent lag between continuum variations in $\mathrm{B}$ and $\mathrm{R}$-band light curves has negligible effect on the BLR size deduced from cross-correlation between the $\mathrm{B}$ band continuum and the $\mathrm{H} \alpha$ line light curves.

\subsection{JAVELIN}

Apart from CCF analysis, we also used the JAVELIN code developed by $\mathrm{Zu}$ et al. (2011) to calculate the time lag between $B$ and $\mathrm{H} \alpha$ as well as the $B$-band and continuum subtracted $R$-band. In JAVELIN, the driving continuum light curve is modeled using a damped random walk (DRW) process, which provides a satisfactory statistical description of the quasar variability (e.g., Kelly et al. 2009). The DRW model is defined by an exponential covariance matrix with two parameters; amplitude and time scale of the variability. First, a top-hat response function representing the BLR response is convolved with the driving continuum resulting in an emission line light curve, which is a shifted, scaled and smoothed version of the driving continuum light curve. The probability distribution of model parameters and the best-fit model are found by maximizing the likelihood function through a Markov Chain Monte Carlo approach. Using $B$-band as the driving continuum light curve, we searched for a lag within a range of -40 to 60 days. An example of the best-fit model light curve and the distribution of the lag obtained from fitting the $B$-band and $\mathrm{H} \alpha$ line light curves using Equation 2 are shown in Fig. 10. The lag distribution shows two peaks; the most prominent one is at 20 days and a secondary peak is at 50 days. Considering that the most prominent peak is at 20 days together with the result obtained from CCF analysis, we obtained a lag of $22.48_{-0.89}^{+0.09}$ days in the observed frame. We summarize the JAVELIN lag results in Table 4 . We found a lag of $\sim 16$ days between $B$-band and continuum subtracted $R$-band light curves.

We note that JAVELIN can over-fit the noise in the data if the error bars are too small. In fact, JAVELIN fit shows spurious peak in the B-band model light curve at MJD 58400 (see Fig. 10) having no observed data at that epoch. Therefore, we did two tests to check the validity of our JAVELIN results; (1) we removed the outlier at MJD 583140 from $\mathrm{H} \alpha$ light curve, and performed the JAVELIN fit, and obtained a lag of $22.52_{-6.01}^{+0.37}$ days in the observed frame, (2) after removing the outlier we also inflated the errorbars by a factor of 3 . The light curves are well fitted with JAVELIN providing a lag of $16.66_{-0.19}^{+5.85}$ days (see Fig. 11) in the observed frame. Both the tests provide a consistent lag within the errorbars, and agree well with the one found from the original light curves. Therefore, the lag measurement using JAVELIN is reliable. The lags obtained by JAVELIN and ICCF are consistent with each other, therefore, in all further discussion, we consider the lags obtained from ICCF analysis. 

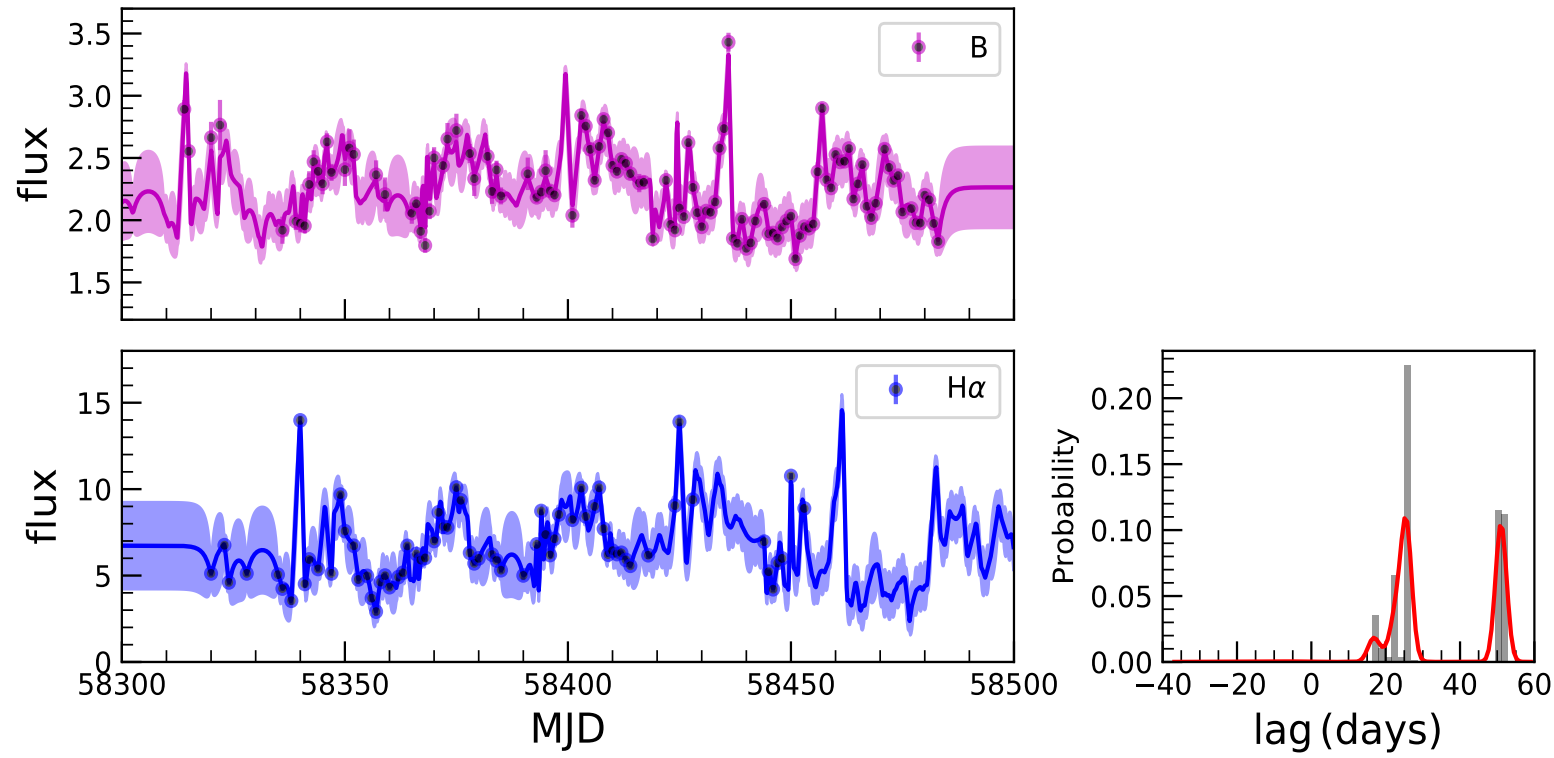

Figure 10. JAVELIN fit to the $\mathrm{B}$ and $\mathrm{H} \alpha$ light curves. The points with errorbars are the observed data while the solid line represents the best fit and shaded region shows $1 \sigma$ error. The lag probability distribution along with smooth kernel density are shown in the lower-right panel.
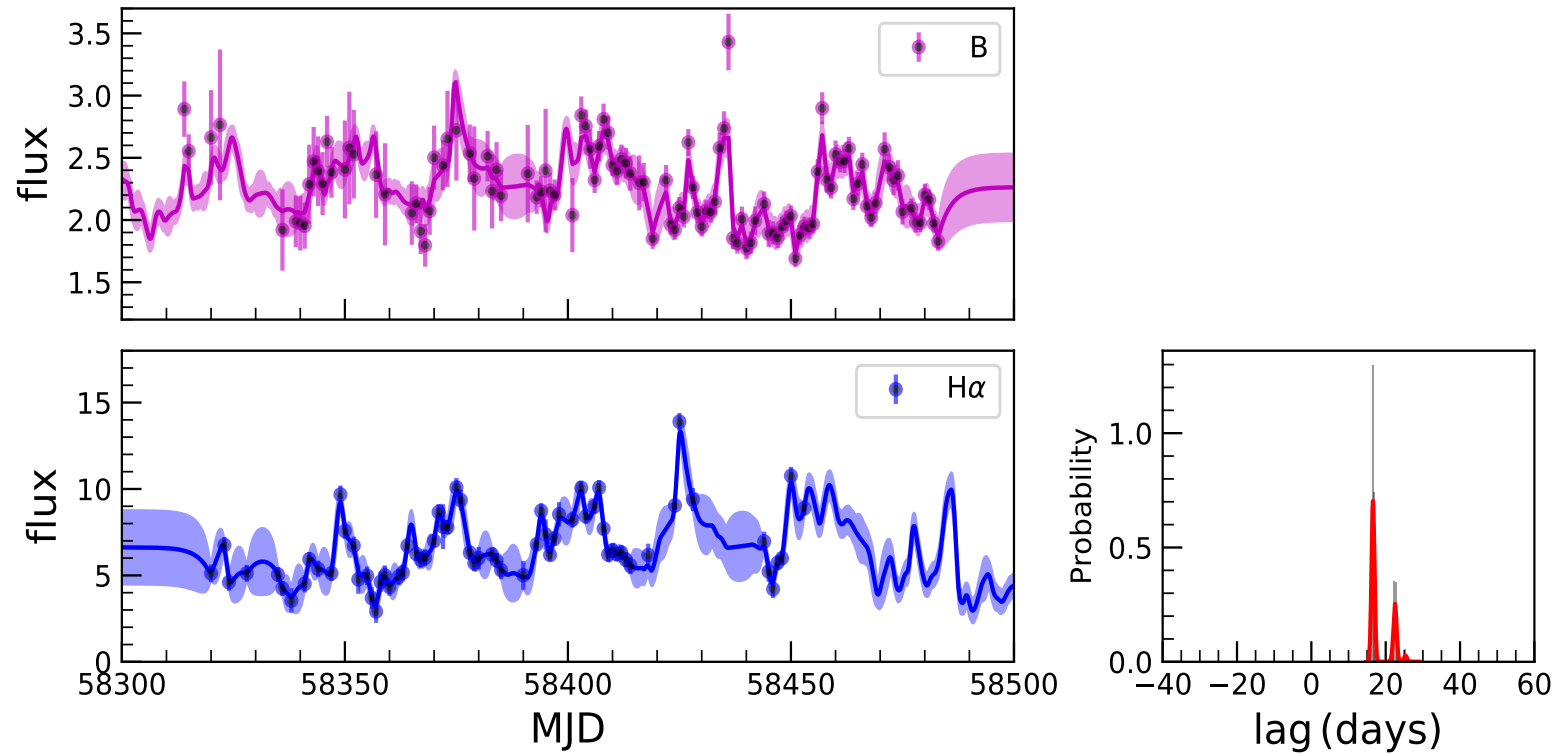

Figure 11. JAVELIN fit to the B and $\mathrm{H} \alpha$ light curves after removing the outlier at MJD 583140 and inflating the errorbars by a factor of 3.

Table 4. Lag measurements. Columns are (1) method used for continuum subtraction (2) filters (3-4) $\tau_{\text {cent }}$ with errors in days as obtained by ICCF and JAVELIN in observer's frame. R-band fluxes were corrected for the continuum using power-law with slope $\alpha$.

\begin{tabular}{llll}
\hline $\begin{array}{l}\text { method } \\
(1)\end{array}$ & $\begin{array}{l}\text { filter } \\
(2)\end{array}$ & $\begin{array}{l}\text { ICCF } \\
(3)\end{array}$ & $\begin{array}{l}\text { JAVELIN } \\
(4)\end{array}$ \\
\hline direct & $\mathrm{B}-\mathrm{H} \alpha$ & $22.00_{-2.17}^{+1.53}$ & $25.42_{-3.03}^{+0.11}$ \\
PL subtracted $(\alpha=1 / 3)$ & $\mathrm{B}-\mathrm{H} \alpha$ & $22.00_{-1.07}^{+0.96}$ & $22.48_{-0.89}^{+0.09}$ \\
PL subtracted $(\alpha=1 / 3)$ & $\mathrm{B}-\mathrm{R}$ & $16.00_{-2.00}^{+0.51}$ & $16.41_{-0.83}^{+0.09}$ \\
\hline
\end{tabular}

\section{DISCUSSION}

From the single-epoch spectrum of Mrk 590 we estimated the monochromatic continuum luminosity at $5100 \AA\left(L_{5100}\right)$ of $43.12 \pm 0.01 \mathrm{erg} \mathrm{s}^{-1}$ (see section 2.2). This corresponds to an expected BLR size of $\sim 12$ days based on the best-fit size-luminosity relation of Bentz et al. (2013), which is a factor of 2 smaller than our measurement. We note that the $L_{5100}$ could be affected by some host galaxy contribution. Although the spectrum does not allow us to clearly separate the host galaxy contribution, photometric image decomposition method provides host galaxy contribution to the total 
flux, which is $\sim 50 \%$ in the observed S II band data. The host galaxy corrected AGN luminosity in $B$-band is found to be $\log L_{\mathrm{AGN}}=43.36 \pm 0.01 \mathrm{erg} \mathrm{s}^{-1}$, which is in excellent agreement with the AGN luminosity of $\log L_{\mathrm{AGN}}=43.31 \pm 0.05$ $\mathrm{erg} \mathrm{s}^{-1}$ at $5100 \AA$ wavelength recently obtained by Kokubo \& Minezaki (2020).

The position of Mrk 590 in the size-luminosity diagram is shown in Fig. 12 based on our H $\alpha$ BLR size measurement and $L_{5100}$ (host uncorrected) along with other AGN from the literature. Our measurement does not significantly deviate from the best-fit relation. Peterson et al. (1998b) performed $\mathrm{H} \beta$ spectroscopic RM of Mrk 590 and estimated a range of BLR size depending on the observational epoch ranging from $14.0_{-8.5}^{+8.5}$ light-days (MJD $\left.=48848-49048\right)$ to $29.2_{-5.0}^{+4.9}$ lightdays (MJD $=49183-49338$ ). Therefore, our measured lag is consistent with the measurement of Peterson et al. (1998b). Note that, our result is based on the $\mathrm{H} \alpha$ lag. Bentz et al. (2010) found that on-average $\mathrm{H} \alpha$ lag is 1.54 times larger than the $\mathrm{H} \beta$ lag. Therefore, the position of Mrk 590 in Fig. 12 is consistent with that expectation.

Our measured BLR size together with other measurements on Mrk 590 are found to be comparable with the inner radius of the dust torus found by Kokubo \& Minezaki (2020). This could be due to two reasons; (a) the DRM observations were carried out during the period $2003-2007$ when the source was in a faint state. As a result, the drastic decrease in the ionizing continuum radiation from the accretion disk causes the dust particles to exist closer to the BLR region making the torus size comparable to the BLR size. According to Kokubo \& Minezaki (2020), after the drastic drop in accretion disk luminosity, the dust replenishment is attained by formation of new dust grains in the BLR/innermost dust torus region, and (b) the distribution of dust particles in the torus region is not uniform as shown in the Unification scheme of AGN and may change over time. Because of the presence of a clumpy dust torus (Stalevski et al. 2013), the obscuration of ionizing continuum by the torus will also vary over time which may cause the torus to lie close to the BLR over a certain period of time. The clumpy dust torus can even play an important role in making an AGN a "changing look AGN" due to the varying obscuration of the emission line fluxes from the BLR along the line of sight over time.

The spectra of Mrk 590 obtained by Peterson et al. (1998b) show clear presence of a broad $\mathrm{H} \beta$ line having an FWHM of $\sim 2300 \mathrm{~km} \mathrm{~s}^{-1}$. However, between $2006-$ 2013 , its continuum luminosity decreased by a factor of 100 and the broad lines disappeared making it a Type 1.9 - 2 AGN (Denney et al. 2014). The MUSE spectra obtained by Raimundo et al. (2019) recently in October 2017 showed strong broad Balmer component confirming the reappearance of the BLR and transition of Mrk 590 from Type 2 to Type 1. Our Subaru spectrum obtained in October 2018 shows both $\mathrm{H} \beta$ and $\mathrm{H} \alpha$ broad-lines with FWHM of $10390 \pm 1718 \mathrm{~km} \mathrm{~s}^{-1}$ and $6478 \pm 240 \mathrm{~km} \mathrm{~s}^{-1}$, respectively. This reconfirms that Mrk 590 is currently in the Type 1 state. Using the measured $\mathrm{H} \alpha$ BLR size of $21.44_{-2.11}^{+1.49}$ lightdays and the FWHM of the $\mathrm{H} \alpha$ line, the black hole mass is found to be $1.96_{-0.21}^{+0.15} \times 10^{8} M_{\odot}$ using virial relation and a scale factor of 1.12 . The black hole mass from the singleepoch spectrum is found to be $(1.59 \pm 0.14) \times 10^{8} M_{\odot}$ based on the $\mathrm{H} \alpha$ line luminosity and the FWHM using the relation given by Woo et al. (2015). Both black hole masses are con-

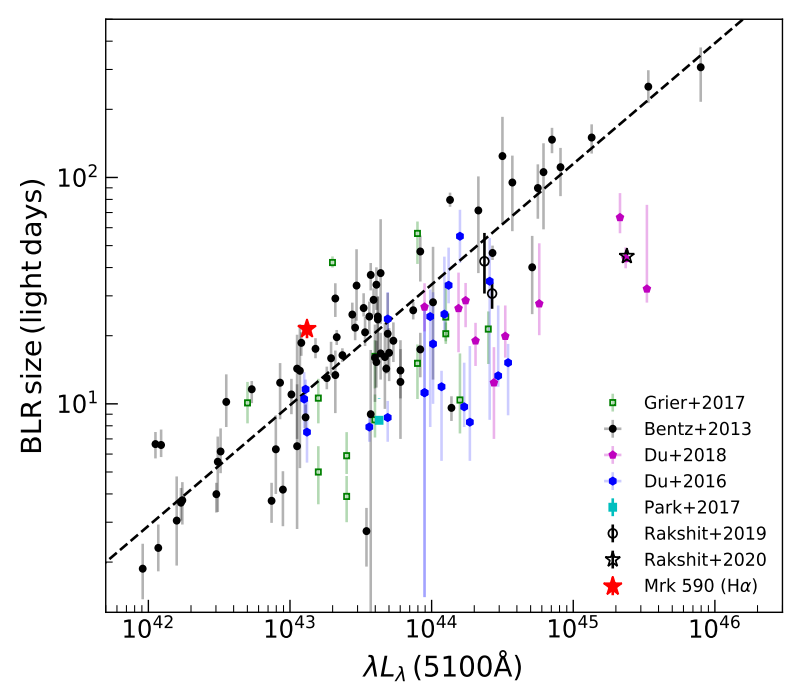

Figure 12. The BLR size-luminosity relation of Mrk 590 along with other AGN from literature (Bentz et al. 2013; Du et al. 2016; Grier et al. 2017; Du et al. 2018; Rakshit \& Woo 2018; Rakshit 2020). The best-fit size-luminosity relation from Bentz et al. (2013) is shown as dotted line.

sistent with each other. Comparing the black hole mass of $(4.75 \pm 0.74) \times 10^{7} \mathrm{M} \odot$ measured by Peterson et al. (2004), our measurement is a factor $\sim 4$ larger, which is due to the increase in the line width of the broad Balmer component by a factor of $\sim 2$. Pozo Nuñez et al. (2014) found that symmetric cutting of emission line wings by the narrow-band filter may lead to over estimation of the BLR size by less than 5 percent. Taking this also into account, our estimated BLR size and black hole mass may have additional uncertainty of not more than 5 percent.

\section{SUMMARY}

We carried out photometric reverberation mapping of Mrk 590 using broad $B$ and $R$ filters, as well as narrow $\mathrm{H} \alpha$ and $\mathrm{S}$ II bands. The narrow S II-filter covers the redshifted $\mathrm{H} \alpha$ line allowing us to construct $\mathrm{H} \alpha$ line light curve and estimate the size of the BLR in Mrk 590 through cross-correlation analysis. Our main results are summarized below.

(i) A significant contribution of the host-galaxy was found in all bands. This contribution was removed using two dimensional image decomposition of the stacked images. The host-corrected luminosity of the AGN in $B, R, \mathrm{H} \alpha$ and S II bands are $\log L_{\mathrm{AGN}}=43.36 \pm 0.01,43.53 \pm 0.01,42.81 \pm 0.06$ and $43.71 \pm 0.01 \mathrm{erg} \mathrm{s}^{-1}$, respectively.

(ii) All the light curves show significant flux variations, which enabled us to carry out time series analysis to estimate the BLR size of Mrk 590. We constructed H $\alpha$ line light curve using two different methods. We corrected the continuum contamination in the narrow S II-band (1) using the observed narrow $\mathrm{H} \alpha$-band data which traces the continuum close to the S II-band and (2) considering a fixed PL slope of $\alpha=1 / 3$ to the observed B-band data. We found no difference in the measured lags between method 1 and 2 both using CCF and JAVELIN analysis.

(iii) The broad $R$-band data also contains the $\mathrm{H} \alpha$ line 
flux. We removed the continuum flux in the observed $R$ band by extrapolating the continuum measured in the $B$ band and assuming an $\alpha$ value of $1 / 3$. We found a lag of $\sim 16$ days between the $B$-band light curve and the continuum subtracted $R$-band light curve $\left(\mathrm{H} \alpha^{\prime \prime}\right)$. The lower value of the lag is due to the larger contribution of the AGN continuum to the broad $R$-band compared to the narrow $\mathrm{S}$ II band.

(iv) Our estimated BLR size based on $\mathrm{H} \alpha$ line in the rest frame of the source is $21.44_{-2.11}^{+1.49}$ days, which is equivalent to 0.018 pc. The H $\alpha$ BLR size of Mrk 590 is consistent with the best-fit size-luminosity relation of AGN.

(v) The Subaru spectrum shows strong $\mathrm{H} \beta$ and $\mathrm{H} \alpha$ lines with FWHM of $6478 \pm 240$ and $10390 \pm 1718 \mathrm{~km} \mathrm{~s}^{-1}$, respectively, suggesting the transition of Mrk 590 from Type 2 (in 2006 - 2013) to Type 1 (in 2017 - 2019). The black hole mass measured from the single-epoch Subaru spectrum is found to be $1.96_{-0.21}^{+0.15} \times 10^{8} M_{\odot}$.

\section{ACKNOWLEDGEMENTS}

We thank the anonymous referee for her/his critical comments that led to the improvement of the manuscript. AKM and RS thank the National Academy of Sciences, India (NASI), Prayagraj for funding and Director, IIA for hosting and providing infrastructural support to this project. AKM also thank the Humboldt foundataion, Germany for the funding to visit ESO, Germany, where part of the reduction of the observed data and analysis were carried out. This research is based in part on data collected at Subaru Telescope, which is operated by the National Astronomical Observatory of Japan. We are honored and grateful for the opportunity of observing the Universe from Maunakea, which has the cultural, historical and natural significance in Hawaii.

\section{DATA AVAILABILITY}

The data underlying this article are available in the article and in its online supplementary material.

\section{REFERENCES}

Andjelić M., Stavrev K., Arbutina B., Ilić D., Urošević D., 2011, Baltic Astronomy, 20, 459

Bentz M. C., Katz S., 2015, PASP, 127, 67

Bentz M. C., Peterson B. M., Netzer H., Pogge R. W., Vestergaard M., 2009, ApJ, 697, 160

Bentz M. C., et al., 2010, The Astrophysical Journal, 716, 993

Bentz M. C., et al., 2013, ApJ, 767, 149

Bessell M. S., Castelli F., Plez B., 1998, A\&A, 333, 231

Blandford R. D., McKee C. F., 1982, ApJ, 255, 419

Boroson T. A., Green R. F., 1992, ApJS, 80, 109

Cellone S. A., Romero G. E., Combi J. A., 2000, AJ, 119, 1534

Denney K. D., et al., 2014, ApJ, 796, 134

Du P., et al., 2014, ApJ, 782, 45

Du P., et al., 2016, ApJ, 825, 126

Du P., et al., 2018, ApJ, 856, 6

Edelson R. A., Krolik J. H., 1988, ApJ, 333, 646

Edelson R., Turner T. J., Pounds K., Vaughan S., Markowitz A., Marshall H., Dobbie P., Warwick R., 2002, ApJ, 568, 610
Edri H., Rafter S. E., Chelouche D., Kaspi S., Behar E., 2012, ApJ, 756, 73

Fausnaugh M. M., et al., 2016, ApJ, 821, 56

Fausnaugh M. M., et al., 2018, ApJ, 854, 107

Fitzpatrick E. L., 1999, PASP, 111, 63

Gaskell C. M., Peterson B. M., 1987, ApJS, 65, 1

Gaskell C. M., Sparke L. S., 1986, ApJ, 305, 175

Gravity Collaboration et al., 2018, Nature, 563, 657

Grier C. J., et al., 2017, ApJ, 851, 21

Hernández Santisteban J. V., et al., 2020, MNRAS, 498, 5399

Homayouni Y., et al., 2021, arXiv e-prints, p. arXiv:2105.02884

Kelly B. C., Bechtold J., Siemiginowska A., 2009, ApJ, 698, 895

Kim M., Ho L. C., Peng C. Y., Barth A. J., Im M., 2017, ApJS, 232,21

Kishimoto M., Hönig S. F., Beckert T., Weigelt G., 2007, A\&A, 476,713

Kishimoto M., Antonucci R., Blaes O., Lawrence A., Boisson C., Albrecht M., Leipski C., 2008, Nature, 454, 492

Kokubo M., Minezaki T., 2020, MNRAS, 491, 4615

Koshida S., et al., 2014, ApJ, 788, 159

LaMassa S., 2015, in Gandhi P., Hoenig S. F., eds, TORUS2015: The AGN Unification Scheme After 30 Years.

Lynden-Bell D., 1969, Nature, 223, 690

Mandal A. K., et al., 2018, MNRAS, 475, 5330

Mandal A. K., et al., 2021, MNRAS, 501, 3905

Martin J. R., et al., 2019, ApJS, 240, 12

Minezaki T., Yoshii Y., Kobayashi Y., Enya K., Suganuma M., Tomita H., Aoki T., Peterson B. A., 2004, ApJ, 600, L35

Noguchi K., et al., 2002, Publications of the Astronomical Society of Japan, 54, 855

Peng C. Y., Ho L. C., Impey C. D., Rix H.-W., 2002, AJ, 124, 266

Peng C. Y., Ho L. C., Impey C. D., Rix H.-W., 2010, AJ, 139, 2097

Peterson B. M., 1993, PASP, 105, 247

Peterson B. M., Horne K., 2004, Astronomische Nachrichten, 325, 248

Peterson B. M., Wanders I., Horne K., Collier S., Alexander T., Kaspi S., Maoz D., 1998a, PASP, 110, 660

Peterson B. M., Wanders I., Bertram R., Hunley J. F., Pogge R. W., Wagner R. M., 1998b, ApJ, 501, 82

Peterson B. M., et al., 2004, ApJ, 613, 682

Pozo Nuñez F., et al., 2014, A\&A, 568, A36

Raimundo S. I., Vestergaard M., Koay J. Y., Lawther D., Casasola V., Peterson B. M., 2019, MNRAS, 486, 123

Rakshit S., 2020, arXiv e-prints, p. arXiv:2007.07672

Rakshit S., Woo J.-H., 2018, ApJ, 865, 5

Rakshit S., Stalin C. S., Kotilainen J., 2020, ApJS, 249, 17

Rani P., Stalin C. S., Rakshit S., 2017, MNRAS, 466, 3309

Runnoe J. C., Brotherton M. S., Shang Z., 2012, MNRAS, 422, 478

Salpeter E. E., 1964, ApJ, 140, 796

Schlegel D. J., Finkbeiner D. P., Davis M., 1998, ApJ, 500, 525

Shakura N. I., Sunyaev R. A., 1973, A\&A, 500, 33

Stalevski M., Fritz J., Baes M., Popovic L. C., 2013, arXiv eprints, p. arXiv: 1301.4244

Suganuma M., et al., 2006, ApJ, 639, 46

Tomita H., et al., 2006, ApJ, 652, L13

Vaughan S., Edelson R., Warwick R. S., Uttley P., 2003, MNRAS, 345,1271

Vincentelli F. M., et al., 2021, MNRAS, 504, 4337

Wang J.-M., et al., 2014, ApJ, 793, 108

Wilhite B. C., Vanden Berk D. E., Kron R. G., Schneider D. P., Pereyra N., Brunner R. J., Richards G. T., Brinkmann J. V., 2005, ApJ, 633, 638

Woo J.-H., Yoon Y., Park S., Park D., Kim S. C., 2015, ApJ, 801, 38 
Zacharias N., Finch C., Frouard J., 2017, VizieR Online Data Catalog, p. I/340

Zu Y., Kochanek C. S., Peterson B. M., 2011, ApJ, 735, 80 


\section{$16 \quad$ Mandal et al.}

This paper has been typeset from a $\mathrm{T}_{\mathrm{E} X} \mathrm{X} \mathrm{LT}_{\mathrm{E}} \mathrm{X}$ file prepared by the author. 